\title{
Surveillance for Violent Deaths - National Violent Death Reporting System, 32 States, 2016
}




\section{CONTENTS}

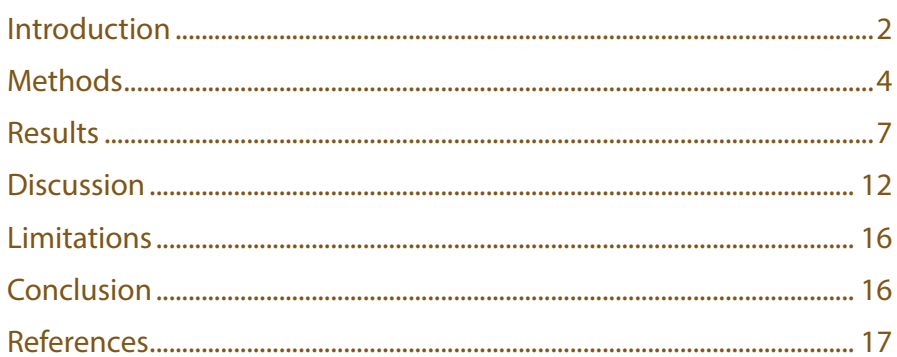

The MMWR series of publications is published by the Center for Surveillance, Epidemiology, and Laboratory Services, Centers for Disease Control and Prevention (CDC), U.S. Department of Health and Human Services, Atlanta, GA 30329-4027.

Suggested citation: [Author names; first three, then et al., if more than six.] [Title]. MMWR Surveill Summ 2019;68(No. SS-\#):[inclusive page numbers].

\section{Centers for Disease Control and Prevention \\ Robert R. Redfield, MD, Director}

Anne Schuchat, MD, Principal Deputy Director

Chesley L. Richards, MD, MPH, Deputy Director for Public Health Science and Surveillance

Rebecca Bunnell, PhD, MEd, Director, Office of Science

Barbara Ellis, PhD, MS, Acting Director, Office of Science Quality, Office of Science

Michael F. Iademarco, MD, MPH, Director, Center for Surveillance, Epidemiology, and Laboratory Services

\section{MMWR Editorial and Production Staff (Serials)}

Charlotte K. Kent, PhD, MPH, Editor in Chief Christine G. Casey, MD, Editor

Mary Dott, MD, MPH, Online Editor

Terisa F. Rutledge, Managing Editor

David C. Johnson, Lead Technical Writer-Editor Marella Meadows, Project Editor

Matthew L. Boulton, MD, MPH

Virginia A. Caine, MD

Katherine Lyon Daniel, $\mathrm{PhD}$

Jonathan E. Fielding, MD, MPH, MBA

David W. Fleming, MD

William E. Halperin, MD, DrPH, MPH
Martha F. Boyd, Lead Visual Information Specialist Maureen A. Leahy, Julia C. Martinroe, Stephen R. Spriggs, Tong Yang, Visual Information Specialists Quang M. Doan, MBA, Phyllis H. King, Terraye M. Starr, Moua Yang,
MMWR Editorial Board

Timothy F. Jones, MD, Chairman

Robin Ikeda, MD, MPH

Phyllis Meadows, PhD, MSN, RN

Jewel Mullen, MD, MPH, MPA

Jeff Niederdeppe, $\mathrm{PhD}$

Patricia Quinlisk, MD, MPH
Stephen C. Redd, MD

Patrick L. Remington, MD, MPH

Carlos Roig, MS, MA

William Schaffner, MD

Morgan Bobb Swanson, BS 


\title{
Surveillance for Violent Deaths - National Violent Death Reporting System, 32 States, 2016
}

\author{
Allison Ertl, $\mathrm{PhD}^{1}$; Kameron J. Sheats, $\mathrm{PhD}^{1}$; Emiko Petrosky, MD ${ }^{1}$; Carter J. Betz, MS ${ }^{1}$; Keming Yuan, MS ${ }^{1}$; Katherine A. Fowler, $\mathrm{PhD}^{1}$ \\ ${ }^{1}$ Division of Violence Prevention, National Center for Injury Prevention and Control, CDC
}

\begin{abstract}
Problem/Condition: In 2016, approximately 65,000 persons died in the United States as a result of violence-related injuries. This report summarizes data from CDC's National Violent Death Reporting System (NVDRS) regarding violent deaths from 32 U.S. states for 2016. Results are reported by sex, age group, race/ethnicity, type of location where injured, method of injury, circumstances of injury, and other selected characteristics.
\end{abstract}

Period Covered: 2016.

Description of System: NVDRS collects data regarding violent deaths obtained from death certificates, coroner/medical examiner reports, law enforcement reports, and secondary sources (e.g., child fatality review team data, Supplementary Homicide Reports, hospital data, and crime laboratory data). This report includes data collected from 32 states for 2016 (Alaska, Arizona, Colorado, Connecticut, Georgia, Hawaii, Illinois, Indiana, Iowa, Kansas, Kentucky, Maine, Maryland, Massachusetts, Michigan, Minnesota, New Hampshire, New Jersey, New Mexico, New York, North Carolina, Ohio, Oklahoma, Oregon, Pennsylvania, Rhode Island, South Carolina, Utah, Vermont, Virginia, Washington, and Wisconsin). NVDRS collates information for each death and links deaths that are related (e.g., multiple homicides, homicide followed by suicide, or multiple suicides) into a single incident.

Results: For 2016, NVDRS captured 40,374 fatal incidents involving 41,466 deaths in the 32 states included in this report. The majority $(62.3 \%)$ of deaths were suicides, followed by homicides $(24.9 \%)$, deaths of undetermined intent $(10.8 \%)$, legal intervention deaths (1.2\%) (i.e., deaths caused by law enforcement and other persons with legal authority to use deadly force acting in the line of duty, excluding legal executions), and unintentional firearm deaths $(<1.0 \%)$. (The term legal intervention is a classification incorporated into the International Classification of Diseases, Tenth Revision [ICD-10] and does not denote the lawfulness or legality of the circumstances surrounding a death caused by law enforcement.) Demographic patterns varied by manner of death. Suicide rates were highest among males, non-Hispanic American Indians/Alaska Natives, non-Hispanic whites, adults aged 45-64 years, and men aged $\geq 75$ years. The most common method of injury was a firearm among males and poisoning among females. Suicides were most often preceded by a mental health, intimate partner, substance abuse, or physical health problem or a recent or impending crisis during the previous or upcoming 2 weeks. Homicide rates were highest among males and persons aged $<1$ year and 15-44 years. Among males, non-Hispanic blacks accounted for most homicides and had the highest rate of any racial/ethnic group. The most common method of injury was a firearm. Homicides were most often precipitated by an argument or conflict, occurred in conjunction with another crime, or for females, were related to intimate partner violence. When the relationship between a homicide victim and a suspected perpetrator was known, the suspect was most frequently an acquaintance/ friend among males and a current or former intimate partner among females. Legal intervention death rates were highest among men aged 20-44 years, and the rate among non-Hispanic black males was three times the rate among non-Hispanic white males. Precipitating circumstances for legal intervention deaths most frequently were an alleged criminal activity in progress, reported use of a weapon by the victim in the incident, a mental health or substance abuse problem (other than alcohol abuse), an argument or conflict, or a recent or impending crisis. Unintentional firearm deaths were more frequent among males, non-Hispanic whites, and persons aged 15-24 years. These deaths most often occurred while the shooter was playing with a firearm and most often were precipitated by a person unintentionally pulling the trigger or mistakenly thinking the firearm was unloaded. Rates of deaths of undetermined intent were highest among males, particularly non-Hispanic black and American Indian/Alaska Native males, and adults aged 25-64 years. Substance abuse, mental health problems, physical health problems, and a recent or impending crisis were the most common circumstances preceding deaths of undetermined intent. In 2016, a total of 3,655 youths aged 10-24 years died by suicide. The majority of these decedents were male, non-Hispanic white, and aged 18-24 years. Most decedents aged $10-17$ years died by hanging/strangulation/suffocation (49.3\%),

Corresponding author: Allison Ertl, Division of Violence Prevention, National Center for Injury Prevention and Control, CDC. Telephone: 404-498-1500; E-mail: moq4@cdc.gov. followed by a firearm $(40.4 \%)$, and suicides among this age group were most often precipitated by mental health, family relationship, and school problems. Most suicides among decedents aged 
18-24 years were by a firearm (46.2\%), followed by hanging/strangulation/suffocation (37.4\%), and were precipitated by mental health, substance abuse, intimate partner, and family problems. A recent crisis, an argument or conflict, or both were common precipitating circumstances among all youth suicide decedents.

Interpretation: This report provides a detailed summary of data from NVDRS for 2016. Suicides rates were highest among nonHispanic American Indian/Alaska Native and white males, whereas homicide rates were highest among non-Hispanic black males. Mental health problems, intimate partner problems, interpersonal conflicts, and acute life stressors were primary precipitating events for multiple types of violent deaths, including suicides among youths aged 10-24 years.

Public Health Action: NVDRS data are used to monitor the occurrence of violence-related fatal injuries and assist public health authorities in the development, implementation, and evaluation of programs and policies to reduce and prevent violent deaths. For example, Utah VDRS data were used to help identify suicide risk factors among youths aged 10-17 years, Rhode Island VDRS suicide data were analyzed to identify precipitating circumstances of youth suicides over a 10-year period, and Kansas VDRS data were used by the Kansas Youth Suicide Prevention Task Force. In 2019, NVDRS expanded data collection to include all 50 states, Puerto Rico, and the District of Columbia. This expansion is essential to public health efforts to reduce violent deaths.

\section{Introduction}

In 2016, violence-related injuries led to approximately 65,000 deaths in the United States (1). Suicide was the 10th leading cause of death overall in the United States and disproportionately affected young and middle-aged populations. Suicide was among the top two leading causes of death for persons aged 10-34 years and among the top four for persons aged 35-54 years. Non-Hispanic American Indian/Alaska Native and non-Hispanic white males were disproportionately affected by suicide.

Homicide was the 16th leading cause of death overall in the United States but disproportionately affected young persons (1). Homicide was the fourth leading cause of death for persons aged 1-14 years and the third leading cause of death for persons aged 15-34 years. Young non-Hispanic black males were disproportionately affected by homicide, which was the leading cause of death for non-Hispanic black males aged 15-34 years and the second leading cause of death for non-Hispanic black males aged $1-4$ and $10-14$ years.

Public health authorities require accurate, timely, and complete surveillance data to better understand and ultimately prevent the occurrence of violent deaths in the United States (2). In 2000, in response to an Institute of Medicine* report noting the need for a national fatal intentional injury surveillance system (3), CDC began planning to implement the National Violent Death Reporting System (NVDRS) (2). The goals of NVDRS are to

- collect and analyze timely, high-quality data for monitoring the magnitude and characteristics of violent deaths at national, state, and local levels;

\footnotetext{
*The name of the Institute of Medicine was changed to the National Academy of Medicine, effective July 1, 2015.
}

- ensure data are disseminated routinely and expeditiously to public health officials, law enforcement officials, policymakers, and the public;

- ensure data are used to develop, implement, and evaluate programs and strategies that are intended to reduce and prevent violent deaths and injuries at national, state, and local levels; and

- build and strengthen partnerships among organizations and communities at national, state, and local levels to ensure that data are collected and used to reduce and prevent violent deaths and injuries.

NVDRS is a state-based active surveillance system that collects data on the characteristics and circumstances associated with violence-related deaths in participating states and territories. Deaths include homicides, suicides, legal intervention deaths (i.e., deaths caused by law enforcement acting in the line of duty and other persons with legal authority to use deadly force, excluding legal executions), unintentional firearm deaths, and deaths of undetermined intent that might have been due to violence. ${ }^{\dagger}$ (The term legal intervention is a classification incorporated into the International Classification of Diseases, Tenth Revision [ICD-10] and does not denote the lawfulness or legality of the circumstances surrounding a death caused by law enforcement.) NVDRS data are used to assist development, implementation, and evaluation of programs and strategies designed to reduce and prevent violent deaths at national, state, and local levels.

Before implementation of NVDRS, single data sources (e.g., death certificates) provided only limited information and few circumstances from which to understand patterns of violent deaths.

\footnotetext{
${ }^{\dagger}$ To be included in NVDRS, deaths of undetermined intent must have some evidence of the possibility that the intent was purposeful, including use of a weapon or other evidence that force was used to inflict the injury. Most commonly, the coroner/medical examiner is unsure whether the death was a suicide or unintentional.
} 
NVDRS fills this surveillance gap by providing more detailed information. NVDRS is the first system to 1) provide detailed information on circumstances precipitating violent deaths, 2) link multiple source documents so that each incident can contribute to the study of patterns of violent deaths, and 3) link multiple deaths that are related (e.g., multiple homicides, suicide pacts, or homicide followed by suicide of the suspected perpetrator).

NVDRS data collection began in 2003 with six participating states (Maryland, Massachusetts, New Jersey, Oregon, South Carolina, and Virginia). Seven states (Alaska, Colorado, Georgia, North Carolina, Oklahoma, Rhode Island, and Wisconsin) began data collection in 2004, three (Kentucky, New Mexico, and Utah) in 2005, two (Ohio and Michigan) in 2010, and 14 (Arizona, Connecticut, Hawaii, Illinois, Indiana, Iowa, Kansas, Maine, Minnesota, New Hampshire, New York, Pennsylvania, Vermont, and Washington) in 2015. Eight states (Alabama, California, Delaware, Louisiana, Missouri, Nebraska, Nevada, and West Virginia), the District of Columbia, and Puerto Rico began data collection in 2017. ${ }^{\S}$ In 2018, NVDRS received funding for nationwide expansion, and the remaining 10 states (Arkansas, Florida, Idaho, Mississippi, Montana, North Dakota, South Dakota, Tennessee, Texas, and Wyoming) began data collection in 2019 (Figure). CDC now provides funding for participation to all 50 states, Puerto Rico, and the District of Columbia.

This report summarizes NVDRS data on violent deaths collected by 32 states in 2016 and highlights the finding that during this time suicide was the second leading cause of death among youths aged 10-24 years (1). During 1999-2016, the unadjusted suicide rate among youths aged $10-17$ years increased approximately $48 \%$, from 3.1 to 4.6 per 100,000 population (1). Among youths aged 18-24 years, the unadjusted suicide rate increased $27.1 \%$, from 11.8 to 15.0 per 100,000 population, during this period (1). The increasing suicide rate among youths warrants more comprehensive understanding of the characteristics and circumstances of these deaths to develop suicide prevention for this population.

Twenty-nine of the participating 32 states collected information on all violent deaths occurring in their state (Alaska, Arizona, Colorado, Connecticut, Georgia, Hawaii, Indiana, Iowa, Kansas, Kentucky, Maine, Maryland, Massachusetts, Michigan, Minnesota, New Hampshire, New Jersey, New Mexico, New York, North Carolina, Ohio, Oklahoma, Oregon, Rhode Island, South Carolina, Utah, Vermont, Virginia, and Wisconsin). Three states (Illinois, Pennsylvania, and Washington) joined NVDRS with plans to collect data on violent deaths in a subset of counties that

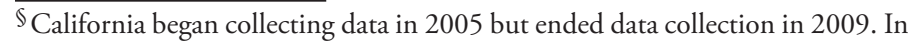
2017, California resumed data collection.
}

FIGURE. States participating in the National Violent Death Reporting System, by year of initial data collection* — United States and Puerto Rico, 2003-2109

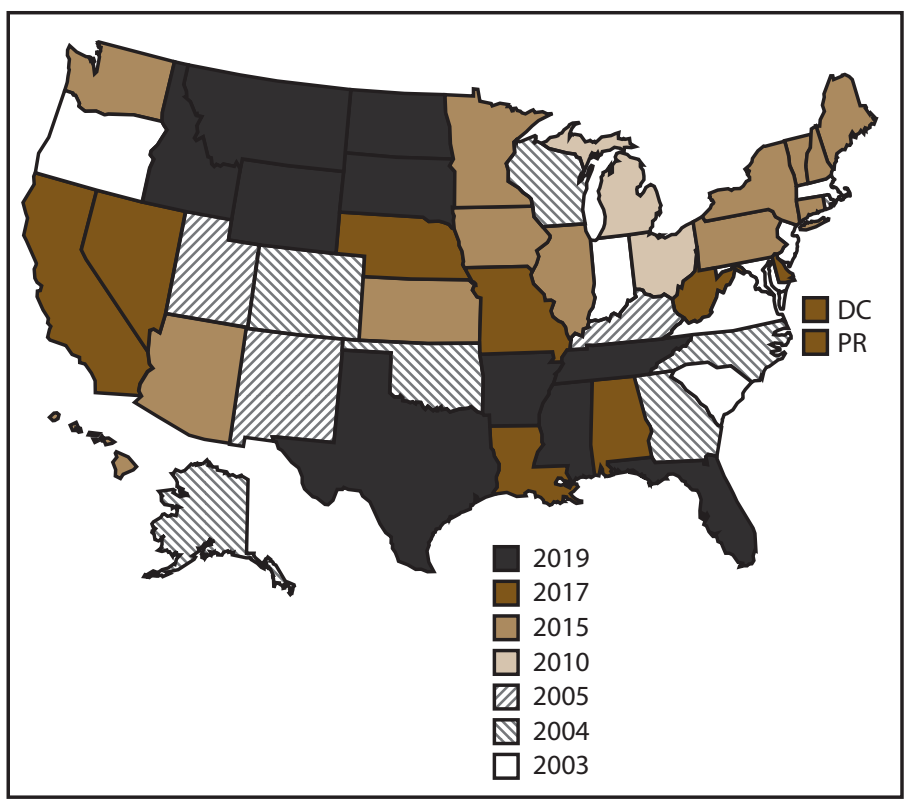

Abbreviations: $\mathrm{DC}=$ District of Columbia; $\mathrm{PR}=$ Puerto Rico.

* California began collecting data for a subset of violent deaths in 2005 but ended data collection in 2009. In 2017, California resumed data collection. Michigan collected data for a subset of violent deaths during 2010-2013 and collected statewide data beginning in 2014. In 2016, Illinois, Pennsylvania, and Washington began collecting data on violent deaths in a subset of counties that represents $\geq 80 \%$ of all violent deaths in the states or in counties where $\geq 1,800$ violent deaths occur.

represents $\geq 80 \%$ of all violent deaths in the state or in counties where $\geq 1,800$ violent deaths occur. In 2016, these states reported data on $\geq 80 \%$ of violent deaths in their state. Because $<100 \%$ of violent deaths were targeted for data collection, data from these three states might not be fully representative of all violent deaths occurring in the state. However, these states were included in the national data set because they captured the majority of violent deaths in their states. In 2016, the 32 states accounted for $58 \%$ of the U.S. population $(1,4)$. NVDRS data are updated annually and are available to the public through CDC's Web-based Injury Statistics Query and Reporting System (WISQARS) at https://www.cdc. gov/injury/wisqars/nvdrs.html. Case-level NVDRS data are available to applicants who meet eligibility requirements via

\footnotetext{
Frequencies and rates of violent deaths included in this report will differ slightly from the frequencies and rates of violent deaths reported by NVDRS WISQARS, which excludes nonresident deaths that occur in participating states (i.e., occurrent deaths). NVDRS tracks both resident and occurrent violent deaths in the overall data set, and the numbers in this report reflect both. States are expected to collect information on violent deaths among their residents, wherever they occur, and fatal violent injuries occurring within their borders irrespective of the decedent's residence status. If the states of residence and injury occurrence are both participating NVDRS states, the state of injury occurrence is responsible for collecting the information. By making this differentiation of responsibility, duplicate reporting is avoided.
} 
access to the NVDRS Restricted Access Database (https://www. cdc.gov/ViolencePrevention/NVDRS/RAD.html).

\section{Methods}

NVDRS compiles information from three required data sources: death certificates, coroner/medical examiner reports, and law enforcement reports. Certain participating states also collect information from secondary sources (e.g., child fatality review team data, Supplementary Homicide Reports, and crime laboratory data). NVDRS combines information for each death and links deaths that are related (e.g., multiple homicides, homicide followed by suicide, or multiple suicides) into a single incident. The ability to analyze linked data can provide more comprehensive understanding of violent deaths. This report presents selected data for 2016. Additional data from 2016 are available (Supplementary Tables, https://stacks. cdc.gov/view/cdc/78990).

In NVDRS, a violent death is defined as a death resulting from the intentional use of physical force or power, threatened or actual, against oneself, another person, or a group or community (5). Information also is collected about unintentional firearm deaths (i.e., a death resulting from a penetrating injury or gunshot wound from a weapon that uses a powder charge to fire a projectile when there was a preponderance of evidence that the shooting was not intentionally directed at the victim) and deaths of undetermined intent (i.e., a death that results from the use of force or power against oneself or another person for which the evidence indicating one manner of death is no more compelling than evidence indicating another). NVDRS cases are coded on the basis of $I C D-10(G)$ or the manner of death assigned by the coroner/medical examiner or law enforcement. Cases are included if they are assigned $I C D-10$ codes (Box 1 ) or a manner of death specified in at least one of the three primary data sources consistent with NVDRS case definitions.

Variables analyzed in NVDRS include

- manner of death (i.e., the intent [homicide, legal intervention, suicide, unintentional, or undetermined] of the person on whom a fatal injury was inflicted);

- mechanism of injury (i.e., the method used to inflict a fatal injury) (Box 2);

- toxicology findings (for decedents who were tested);

- circumstances preceding injury (i.e., the events that preceded and were identified by investigators as relevant and therefore might have contributed to the infliction of a fatal injury) (Box 3);

- whether the decedent was a victim (i.e., a person who died as a result of a violence-related injury) or both a suspect and a victim (i.e., a person believed to have inflicted a fatal injury on a victim who then was fatally injured, such as the perpetrator of a homicide-suicide);

- information about any known suspects (i.e., a person or persons believed to have inflicted a fatal injury on a victim);

- incident (i.e., an occurrence in which one or more persons sustained a fatal injury that was linked to a common event or perpetrated by the same suspect or suspects during a 24-hour period); and

- type of incident (i.e., a combination of the manner of death and the number of victims in an incident).

NVDRS is an incident-based system, and all decedents associated with a given incident are grouped in one record. Decisions about whether two or more deaths are related and belong to the same incident are made on the basis of the timing of the injuries rather than on the timing of the deaths. Deaths resulting from injuries that occur within 24 hours of each other and are clearly linked by source documents (discussed under Manner of Death) would be considered part of the same incident. Examples of an incident include 1) a single isolated violent death, 2) two or more related homicides (including legal intervention deaths) when the fatal injuries were inflicted $<24$ hours apart, 3) two or more related suicides or deaths of undetermined intent when the fatal injuries were inflicted $<24$ hours apart, and 4) a homicide followed by a suicide when both fatal injuries were inflicted $<24$ hours apart ( 7 ).

Information collected from each data source is entered into the NVDRS web-based data entry system (2). This system streamlines data abstraction by allowing abstractors to enter data from multiple sources into the same incident record. Internal validation checks, hover-over features that define selected fields, and other quality control measures are included. Primacy rules and hierarchal algorithms related to the source documents occur at the state level. CDC provides access to the web-based system to each state, district, and territory. State project personnel are provided ongoing coding training to learn and adhere to CDC guidance regarding coding of all variables and technical assistance to help increase data quality. Data are transmitted continuously via the web to a CDC-based server. No personally identifiable information is shared with CDC.

\section{Manner of Death}

A manner (i.e., intent) of death for each decedent is assigned by a trained abstractor who integrates information from all source documents. The abstractor-assigned manner of death must agree with at least one required data source; typically, all source documents are consistent regarding the manner of death. When a discrepancy exists, the abstractor must assign a manner of death on the basis of a preponderance of evidence in the source documents; however, such occurrences are rare (G). 
For example, if two sources report a death as a suicide and a third reports it as a death of undetermined intent, the death is coded as a suicide.

NVDRS data are categorized into five abstractor-assigned manners of death: 1) suicide, 2) homicide, 3) unintentional firearm, 4) undetermined intent, and 5) legal intervention.

- Suicide. A suicide is a death of a person aged $\geq 10$ years resulting from the use of force against oneself when a preponderance of evidence indicates that the use of force was intentional. This category also includes the following scenarios: 1) deaths of persons who intended only to injure rather than kill themselves; 2) persons who initially intended to kill themselves, changed their minds, but died as a result of the acts; 3) deaths associated with risk-taking behavior without clear intent to inflict fatal self-injury but associated with high risk for death (e.g., playing Russian roulette); 4) suicides that occurred while under the influence of substances or drugs that were taken voluntarily; 5) suicides that occurred while under the influence of a mental illness (e.g., experiencing an acute episode of mental illness); and 6) suicides involving another person providing only passive assistance to the decedent (e.g., supplying the means or information needed to complete the act). This category does not include deaths caused by chronic or acute substance abuse without the intent to die, deaths attributed to autoerotic behavior (e.g., self-strangulation during sexual activity), or assisted suicides (legal or nonlegal). Corresponding ICD-10 codes included in NVDRS are X60-X84, Y87.0, and U03 (Box 1).

- Homicide. A homicide is a death resulting from the use of physical force or power, threatened or actual, against another person, group, or community when a preponderance of evidence indicates that the use of force was intentional. Two special scenarios that CDC's National Center for Health Statistics (NCHS) regards as homicides are included in the NVDRS case definition: 1) arson with no specified intent to injure someone and 2) a stabbing with intent unspecified. This category also includes the following scenarios: 1) a death when the suspect intended to only injure rather than kill the victim; 2) a death resulting from a heart attack induced when the suspect used force or power against the victim; 3) a death that occurs when a person kills an attacker in self-defense; 4) a death resulting from a weapon that discharges unintentionally while being used to control or frighten a victim; 5) a death attributed to child abuse without intent being specified; 6) a death attributed to an intentional act of neglect by one person against another; 7) a death of an infant that resulted from a direct injury due to violence sustained before birth, and 8) a death identified as justifiable homicide when the person committing homicide was not a law enforcement officer. This category excludes vehicular homicides without intent to injure, unintentional poisoning deaths due to illegal or prescription drug overdose even when a person who provided drugs was charged with homicide, unintentional firearm deaths (a separate category in NVDRS), combat deaths or acts of war, deaths of unborn fetuses, and deaths of infants that resulted indirectly from violence sustained by the mother before birth (e.g., death from prematurity following premature labor brought on by violence). Corresponding ICD-10 codes included in NVDRS are X85-X99, Y00-Y09, Y87.1, and U01-U02 (Box 1).

- Unintentional firearm. An unintentional firearm death is a death resulting from a penetrating injury or gunshot wound from a weapon that uses a powder charge to fire a projectile and for which a preponderance of evidence indicates that the shooting was not directed intentionally at the decedent. Examples include the following: 1) a person who dies as a result of a celebratory firing that was not intended to frighten, control, or harm anyone; 2) a person who unintentionally shoots himself when using a firearm to frighten, control, or harm another person; 3) a soldier who is shot during a field exercise but not in a combat situation; 4) a person who received a self-inflicted wound while playing with a firearm; 5) a person who mistakenly believes a gun is unloaded and shoots another person; 6) a child aged $<6$ years who shoots himself or another person; and 7) a child who dies after birth from an unintentional firearm injury that was sustained in utero. This category excludes injuries caused by unintentionally striking a person with the firearm (e.g., hitting a person on the head with the firearm rather than firing a projectile) and unintentional injuries from nonpowder guns (e.g., $\mathrm{BB}$, pellet, or other compressed air-powered or gaspowered guns). Corresponding $I C D-10$ codes included in NVDRS are W32-W34 and Y86 (Box 1).

- Undetermined intent. A death of undetermined intent is a death resulting from the use of force or power against oneself or another person for which the evidence indicating one manner of death is no more compelling than evidence indicating another. This category includes coroner/medical examiner rulings (e.g., accident or suicide, undetermined, jumped or fell, or self-inflicted injury or undetermined intent) where records from data providers indicate that investigators did not find enough evidence to determine whether the injury was intentional. Corresponding ICD-10 codes included in NVDRS are Y10-Y34, Y87.2, and Y89.9 (Box 1). 
- Legal intervention. A death from legal intervention is a death in which a person is killed or died as a result of injuries inflicted by a law enforcement officer or other peace officer (i.e., a person with specified legal authority to use deadly force), including military police, while acting in the line of duty. The term legal intervention is a classification from ICD-10 (Y-35.0) and does not denote the lawfulness or legality of the circumstances surrounding the death. Legal intervention deaths also include a small subset of cases in which force was applied without clear lethal intent (e.g., during restraint or when applying force with a typically nondeadly weapon, such as a Taser) or in which the death occurred while the person was fleeing capture. This category excludes legal executions. Corresponding ICD-10 codes included in NVDRS are Y35.0-Y35.4, Y35.6, Y35.7, and Y89.0 (Box 1).

\section{Variables Analyzed}

NVDRS collects approximately 600 unique variables for each death. The number of variables recorded for each incident depends on the content and completeness of the source documents. Variables include manner of death; demographic information; ICD-10 cause of death codes and text descriptors; location, date, and time of injury and death; toxicology results; bodily injuries; precipitating circumstances; victim-suspect relationship and other suspect characteristics; and method of injury (Boxes 1, 2, and 3).

\section{Circumstances Preceding Death}

Circumstances preceding death are defined as the precipitating events that contributed to the infliction of a fatal injury (Box 3). The circumstances are reported on the basis of the content of coroner/medical examiner and law enforcement investigative reports. Certain circumstances are coded to a specific manner of death (e.g., suicide or death of undetermined intent); other circumstances are coded across all manners of death. The data abstractor selects from a list of potential circumstances and is required to code all circumstances that are known to relate to each incident. If circumstances are not known (e.g., for a body found in the woods with no other details reported), the data abstractor leaves the circumstances known variable blank; these deaths are excluded from the denominator for circumstance values. If either the coroner/medical examiner report or law enforcement report indicates the presence of a circumstance, then the abstractor endorses the circumstance (e.g., if the law enforcement report indicated that a decedent had disclosed an intent to die by suicide, then disclosed suicidal intent is endorsed).

\section{Coding Training and Quality Control}

Ongoing coding support for data abstractors is provided through a help desk, monthly conference calls, annual in-person meetings that include coding training for data abstractors, monthly coding work group calls in which all state data abstractors are invited to participate, and regular conference calls with individual states. States also can conduct additional abstractor training workshops and activities at their own discretion, including the use of NVDRS data abstractor e-learning training modules. An NVDRS coding manual ( 7 ) with CDC-issued standard guidance on coding criteria and examples for each data element is provided. Software features to enhance coding reliability include automated validation rules and a hover-over feature containing variable-specific information.

States are requested to perform annual blind reabstractions of a subset of cases using multiple abstractors to identify inconsistencies. Before releasing data each year, CDC also runs a quality control analysis in which multiple variables are reviewed for their appropriateness, with special focus on abstractor-assigned variables (e.g., method and manner of death). If CDC finds inconsistencies, the state is notified and asked for a response or correction. To ensure no duplicate records are in the final data set, NVDRS first uses SAS (version 9.4; SAS Institute) to search for any instances of duplicates of a unique identification variable associated with each decedent record. As a second and final check for duplicates, the SAS data set is created with an index that only executes successfully if no duplicates of this identification variable are found.

\section{Time Frame}

States are required to begin entering all deaths into the webbased system within 6 months from the date the violent death occurred. States then have an additional 18 months from the end of the calendar year in which the violent death occurred to complete each incident record. Although states typically meet these requirements, additional details sometimes arrive after a deadline has passed. New incidents also might be identified after the deadline (e.g., a death certificate is revised, new evidence is obtained that changes a manner of death, or an ICD-10 miscoding is corrected to meet NVDRS inclusion criteria). These additional data are incorporated into NVDRS. Analysis files are updated in real time in the web-based system. On the basis of a recent examination of the past 10 data years, $\mathrm{CDC}$ estimates that case counts are not likely to increase $>1 \%$ after the 18-month data collection period.

\section{Fatal Injuries in 2016}

This report provides data on fatal injuries meeting the NVDRS case definition for violent deaths in 2016 that 
were received by CDC as of September 24, 2018. The 32 participating states used vital statistics death certificate files or coroner/medical examiner reports to identify violent deaths meeting NVDRS case definitions. Each state reported violent deaths of residents that occurred within the state and those of nonresidents for whom a fatal injury occurred within the state (i.e., occurrent deaths). When a violent death was identified, NVDRS data abstractors linked source documents, linked deaths within each incident, coded data elements, and wrote brief narratives of the incident. State data quality was also evaluated by CDC. States meeting minimum or higher data quality standards, such as having descriptive and circumstance data for a sufficient percentage of cases, are included in the NVDRS analytic data set used for this and all other analyses using NVDRS data. All eligible states met this threshold for 2016. State-level data were then consolidated and analyzed.

Numbers, percentages, and crude rates are presented in aggregate for all deaths by abstractor-assigned manner of death. Rates for cells with frequency $<20$ are not reported because of the instability of those rates (7). Illinois, Pennsylvania, and Washington collected data on $\geq 80 \%$ of violent deaths in their state, in accordance with requirements under which the state was funded. Denominators for the rates for these three states represent only the populations of the counties from which data were collected. Rates could not be calculated for certain variables (e.g., precipitating circumstances) because denominators were unknown. Bridged-race 2016 population estimates were used as denominators in the crude rate calculations (8). For compatible numerators for rate calculations to be derived, records listing multiple races were recoded to a single race, when possible, using race-bridging methods described by NCHS (available at https://www.cdc. gov/nchs/nvss/bridged_race.htm) (9).

\section{Results}

\section{All Deaths Captured by NVDRS}

The 32 NVDRS states included in this report collected data on 40,374 incidents and 41,466 deaths that occurred in 2016. Suicides ( $\mathrm{n}=25,850 ; 62.3 \%)$ accounted for the highest rate of violent deaths ( 15.7 per 100,000 population aged $\geq 10$ years), followed by homicides ( $\mathrm{n}=10,336 ; 24.9 \%)(5.5$ per 100,000 population). Deaths of undetermined intent ( $\mathrm{n}=4,470 ; 10.8 \%)$, legal intervention deaths $(\mathrm{n}=515 ; 1.2 \%)$, and unintentional firearm deaths $(\mathrm{n}=295 ;<1.0 \%)$ occurred at lower rates $(2.4$, 0.3 , and 0.2 per 100,000 population, respectively).

\section{Suicides}

\section{Sex, Race/Ethnicity, and Age Group}

In 2016, the 32 NVDRS states included in this report collected data on 25,809 incidents involving suicides, which included 25,850 suicide deaths among persons aged $\geq 10$ years. Overall, the suicide rate was 15.7 per 100,000 population. The overall rate for males was 3.5 times the rate for females (24.8 and 7.0 per 100,000 population, respectively) (Table 1 ); however, the rate for males ranged from 1.9 to 15.9 times the rate for females across age groups and 2.6 to 4.2 times the rate for females across racial/ethnic groups. Adults aged $45-54$ years and 55-64 years (19.7 and 18.3 per 100,000 population, respectively) had the highest rate of suicides across age groups. Youths aged $10-19$ years accounted for $<6 \%$ of all suicides and had the lowest rate among all age groups; however, suicide was the second leading cause of death for this age group in 2016 (1). Non-Hispanic whites accounted for the majority (82.8\%) of suicides. Non-Hispanic American Indians/Alaska Natives had the highest rate of suicides (28.6 per 100,000 population).

Among males, half (50.4\%) of suicide decedents were adults aged 35-64 years. Men aged $\geq 85$ years had the highest rate, followed by men aged $75-84$ and $45-54$ years $(44.5,33.6$, and 29.3 per 100,000 population, respectively) (Table 1 ). NonHispanic American Indian/Alaska Native males had the highest rate of suicides ( 42.8 per 100,000 population), followed by nonHispanic white males (29.7 per 100,000 population). The rate of suicide for non-Hispanic American Indian/Alaska Native males was approximately 3.5 times the rate for males with the lowest rate, Asians/Pacific Islanders (12.1 per 100,000 population).

Among females, women aged 35-64 years also accounted for the majority $(58.7 \%)$ of suicides. Women aged $45-54$ years had the highest rate of suicide (10.4 per 100,000 population). Rates were highest for non-Hispanic American Indian/Alaska Native (15.1 per 100,000 population) and non-Hispanic white (8.4 per 100,000 population) females and lowest for nonHispanic black (3.0 per 100,000 population) and Hispanic (3.4 per 100,000 population) females.

\section{Method and Location of Injury}

Firearms were used in approximately half $(49.4 \%)$ of suicides, followed by hanging/strangulation/suffocation $(27.8 \%)$ and poisoning $(14.4 \%)(7.8,4.4$, and 2.3 per 100,000 population, respectively); the remaining methods used accounted for $8.4 \%$ of suicides (Table 1 ). Among males, the most common method used was a firearm (55.3\%), followed by hanging/strangulation/suffocation (27.7\%). Among females, poisoning (32.9\%), a firearm (29.1\%), and hanging/ strangulation/suffocation (28.2\%) were used in approximately equal proportions. The most common place of suicide was a 
house/apartment (74.0\%) for both males and females $(72.3 \%$ and $79.8 \%$, respectively), followed by a natural area (5.1\%), a motor vehicle $(5.0 \%)$, a street/highway $(2.3 \%)$, and a hotel/ motel $(2.2 \%)$.

\section{Toxicology Results of Decedent}

Tests for alcohol were conducted for $51.5 \%$ of suicide decedents (Table 2). Tests for amphetamines, antidepressants, benzodiazepines, cocaine, marijuana, and opioids were conducted for $36.5 \%, 27.7 \%, 39.0 \%, 37.7 \%, 32.0 \%$, and $40.7 \%$ of decedents, respectively. Among those with positive results for alcohol (40.2\%), 64.4\% of those who tested positive had a blood alcohol concentration $(B A C) \geq 0.08 \mathrm{~g} / \mathrm{dL}$. Results for opioids (including illicit and prescription drugs) were positive in $27.5 \%$ of decedents tested for these substances. Results for amphetamines, cocaine, and marijuana were positive in $11.3 \%, 6.7 \%$, and $23.2 \%$ of decedents tested, respectively. Of those tested for antidepressants, $40.1 \% \mathrm{had}$ positive results at the time of their death, and $30.9 \%$ of those tested for benzodiazepines had positive results. Carbon monoxide was tested for in a substantially smaller proportion of decedents $(7.7 \%)$ but was identified in approximately one third of those decedents (30.7\%).

\section{Precipitating Circumstances}

Precipitating circumstances were known for 23,630 (91.4\%) of suicide decedents (Table 3). Overall, mental health problems were the most common circumstance, with $49.0 \%$ of decedents described as having a current diagnosed mental health problem, $36.9 \%$ as experiencing a depressed mood at the time of their death, and $27.0 \%$ as currently receiving mental health treatment. Among the 11,577 decedents with a current diagnosed mental health problem, depression/ dysthymia (74.0\%), anxiety disorder (18.4\%), and bipolar disorder (14.2\%) were the most common diagnoses.

Alcohol or other substance abuse problems were reported for $17.8 \%$ and $16.8 \%$ of suicide decedents, respectively (Table 3 ). A crisis during the previous or upcoming 2 weeks (31.2\%), intimate partner problems (28.1\%), physical health problems $(22.3 \%)$, and an argument or conflict (16.2\%) were among the most commonly reported precipitating circumstances. Among other circumstances related to the suicide event, $33.6 \%$ of decedents left a suicide note, $32.6 \%$ had a history of suicidal thoughts or plans, $20.3 \%$ had a history of previous suicide attempts, and $23.5 \%$ had disclosed suicidal intent to another person. Of those who disclosed intent, the greatest proportion of disclosures was to a previous or current intimate partner $(37.8 \%)$, followed by a family member other than an intimate partner $(29.5 \%)$.
When examining known circumstances by sex, a greater percentage of female decedents was reported to have had a current diagnosed mental health problem $(64.3 \%)$ than male decedents (44.3\%). Similar percentages of male and female suicide decedents were reported to have had a depressed mood at the time of death $(37.2 \%$ and $36.1 \%$, respectively). A greater percentage of female $(40.2 \%)$ than male $(23.0 \%)$ decedents was known to have been receiving mental health treatment at the time of death. Suicide events, including leaving a suicide note, history of suicidal thoughts and plans, and history of suicide attempts, were reported more frequently for females than males (Table 3).

\section{Homicides}

\section{Sex, Race/Ethnicity, and Age Group}

The 32 NVDRS states included in this report collected data on 9,692 incidents involving homicides, which included 10,336 homicide deaths in 2016 . Overall, the homicide rate was 5.5 per 100,000 population.

Homicide rates for males aged 15-24 years ranged from 5.4 to 6.8 times the rate for females across age groups; however, rates were similar for males and females among persons aged $<1-14$ years and $\geq 85$ years (Table 4 ). Non-Hispanic black males accounted for $61.2 \%$ of homicides among males and approximately half $(\mathrm{n}=5,010 ; 48.5 \%)$ of all homicides and had the highest rate of homicide across any racial/ethnic group (41.5 per 100,000 population). This rate was 13.8 times the homicide rate for non-Hispanic white males (3.0 per 100,000 population), 2.7 times the homicide rate for American Indian/ Alaska Native males (15.4 per 100,000 population), and approximately 5.0 times the rate for Hispanic males (8.6 per 100,000 population). The homicide rate for infants aged $<1$ year was 3.3 times the rate for children aged $1-4$ years $(7.0$ and 2.1 per 100,000 population, respectively). Among female homicide decedents, the rates were highest among infants aged $<1$ year (7.0 per 100,000 population) and non-Hispanic blacks (5.9 per 100,000 population). The homicide rate for non-Hispanic black females (5.9 per 100,000 population) was 3.7 times the rate for non-Hispanic white females (1.6 per 100,000 population), 5.4 times the rate for Asian/ Pacific Islander females (1.1 per 100,000 population), and approximately 3.0 times the rate for Hispanic females $(1.9$ per 100,000 population).

\section{Method, Location of Injury, and Victim-Suspect Relationship}

Firearms were used in $73.8 \%$ of homicides, followed by sharp instruments (10.6\%), personal weapons (e.g., hands, feet, or fists; $4.1 \%$ ), blunt instruments (4.0\%), and hanging/ 
strangulation/suffocation (2.6\%) (Table 4). No other method was used in $>1 \%$ of homicides. Firearms were the most common method used in homicides of both males and females $(78.8 \%$ and $54.4 \%$, respectively); however, the firearm homicide rate for males was 5.8 times the rate for women (7.0 and 1.2 per 100,000 population, respectively). A greater percentage of fatal injuries among females than among males was caused by sharp instruments (15.7\% and $9.3 \%$, respectively), personal weapons $(6.2 \%$ and $3.5 \%$, respectively), blunt instruments (6.5\% and $3.3 \%$, respectively), and hanging/strangulation/ suffocation ( $7.7 \%$ and $1.3 \%$, respectively). A house/apartment was the most common location of homicide (47.3\%), followed by a street/highway $(24.2 \%)$, a motor vehicle $(9.0 \%)$, and a parking lot/public garage/public transport (4.1\%). A greater proportion of homicides of females than males occurred at a house/apartment $(69.3 \%$ and $41.6 \%$, respectively), whereas a greater proportion of homicides of males than females occurred on a street/highway (28.2\% and $8.9 \%$, respectively).

The relationship of the victim to the suspect was known for $41.8 \%$ of homicides. When the relationship was known, the suspect was most often an acquaintance/friend (26.9\%), a current or former spouse/intimate partner $(21.8 \%)$, other person known to the victim (14.4\%), or a stranger (13.0\%). Half of female decedents with information on the perpetrator were killed by a current or former intimate partner (50.1\%); in contrast, only $7.5 \%$ of males were killed by a current or former intimate partner. Among male decedents, the suspect was most frequently an acquaintance/friend (35.2\%).

\section{Precipitating Circumstances}

Precipitating circumstances were identified for $79.3 \%$ of homicides (Table 5). Approximately one in three homicides with known circumstances was precipitated by an argument or conflict (32.1\%). Homicides were commonly precipitated by another crime (31.0\%); in $56.6 \%$ of those cases, the crime was in progress at the time of the incident. The types of crime most frequently precipitating the homicide were assault/homicide $(43.8 \%)$, robbery $(34.6 \%)$, drug trade** $(12.5 \%)$, burglary $(11.9 \%)$, motor vehicle theft $(3.4 \%)$, rape/sexual assault (2.4\%), and arson (2.2\%) (Supplementary Table S7, https:// stacks.cdc.gov/view/cdc/78990). A physical fight between two persons (13.0\%) and drug involvement $(12.0 \%)$ were other common precipitating circumstances. In $15.6 \%$ of homicides with known circumstances, intimate partner violence (IPV) was identified as a contributing factor (Table 5).

Among all reported homicide circumstances, IPV accounted for the largest percentage difference by sex. IPV was a known circumstance for approximately $43.2 \%$ of homicides among

\footnotetext{
** Drug trade is defined as the buying, selling, or passing of drugs in exchange for goods or money. Drug involvement includes drug use in addition to drug trade.
}

females but only $7.5 \%$ of homicides among males (Table 5). An argument or conflict was a factor in $33.1 \%$ of homicides among males and $28.7 \%$ of homicides among females. Physical fights precipitated $14.6 \%$ of homicides among males but only $7.4 \%$ among females. Similarly, drug involvement more commonly precipitated homicide among males, contributing to $13.8 \%$ of homicides among males and $5.8 \%$ among females. Approximately one in 10 homicides of females resulted from caretaker abuse or neglect (10.3\%) compared with only $2.9 \%$ of homicides among males. Gang-related homicides were more common among males (8.6\%) than females (3.2\%). Male decedents used a weapon during the incident in $6.9 \%$ and female decedents in $1.4 \%$ of homicides with known circumstances.

\section{Legal Intervention Deaths}

\section{Sex, Race/Ethnicity, and Age Group}

The 32 NVDRS states included in this report collected data on 510 legal intervention death incidents, which included 515 deaths in 2016. Almost all legal intervention deaths occurring in 2016 were among males (95.5\%) (Table 6). The highest rate was among males aged $30-34$ years $(1.3$ per 100,000 population), followed by those aged $25-29$ years (1.2 per 100,000 population), $35-44$ years (1.1 per 100,000 population), and $20-24$ years (0.9 per 100,000 population). Non-Hispanic white males accounted for half of legal intervention deaths; however, non-Hispanic black males had the highest rate (1.1 per 100,000 population), 2.8 times the rate for non-Hispanic white males $(0.4$ per 100,000$)$. Of the 492 male decedents, $15.4 \%$ were Hispanic; their legal intervention death rate was 0.7 per 100,000 population.

\section{Method and Location of Injury}

Firearms were used in the majority $(96.1 \%)$ of legal intervention deaths (Table 6). Legal intervention deaths occurred most frequently in a house/apartment $(38.4 \%)$, followed by a street/highway $(28.3 \%)$ or a motor vehicle $(9.5 \%)$.

\section{Precipitating Circumstances}

Precipitating circumstances were identified for $98.1 \%$ of legal intervention deaths (Table 7). Approximately $90 \%$ of legal intervention deaths were precipitated by another serious crime. Among those precipitated by another serious crime $(\mathrm{n}=453)$, assault/homicide $(57.0 \%)$, robbery $(11.0 \%)$, motor vehicle theft $(7.5 \%)$, burglary $(4.2 \%)$, and drug trade $(1.8 \%)$ were the types of crimes most frequently precipitating the death (Supplementary Table S9, https://stacks.cdc.gov/ view/cdc/78990). In two thirds (65.8\%) of legal intervention deaths that were precipitated by another crime, a serious crime was reportedly in progress at the time of the incident; in the 
remaining cases, a serious crime might have occurred before the incident (Table 7). The decedent reportedly used a weapon in $72.9 \%$ of cases. In $25.7 \%$ of legal intervention deaths with known circumstances, a substance abuse problem (other than alcohol) was reported as a contributing factor, and $21.0 \%$ of decedents reportedly had a current diagnosed mental health problem. An argument or conflict (14.1\%), being a perpetrator of interpersonal violence during the past month $(9.7 \%)$, family relationship problems (6.9\%), and drug involvement (5.7\%) were other notable precipitating circumstances. Among legal intervention deaths with known circumstances, IPV was identified as a contributing factor in $8.9 \%$ of deaths and a crisis during the previous or upcoming 2 weeks was cited in $15.2 \%$.

\section{Unintentional Firearm Deaths}

\section{Sex, Race/Ethnicity, and Age Group}

The 32 NVDRS states included in this report collected data on 293 incidents involving 295 unintentional firearm injury deaths in 2016. Approximately half ( $\mathrm{n}=151 ; 51.2 \%)$ of these deaths were self-inflicted, and 104 deaths (35.3\%) were known to be inflicted by another person; for the remaining 40 deaths (13.6\%), the person who inflicted the injury was not known (data not reported). Males accounted for $86.4 \%$ of decedents (Table 8 ). Persons aged $\leq 24$ years accounted for more than half $(51.9 \%)$ of all unintentional firearm injury deaths. Approximately $17 \%$ of decedents were aged $<15$ years. The majority of decedents were non-Hispanic whites (59.3\%), followed by non-Hispanic blacks (29.2\%).

\section{Location of Injury and Firearm Type}

Of all unintentional firearm deaths, $73.9 \%$ occurred in a house/apartment, followed by a natural area $(8.8 \%)$ or a motor vehicle (4.4\%) (Table 8$)$. The majority of unintentional firearm injury deaths involved a handgun $(63.1 \%)$, a rifle $(10.8 \%)$, or a shotgun $(8.5 \%)$. In $17.3 \%$ of unintentional firearm deaths, the firearm type was unknown.

\section{Context of Injury and Associated Circumstances}

The context of the injury or associated circumstances was known for $90.8 \%$ of unintentional firearm deaths (Table 9). Overall, the most common context of injury was playing with a gun $(34.7 \%)$, followed by showing the gun to others $(12.3 \%)$, cleaning the gun $(10.1 \%)$, and hunting $(7.8 \%)$. Unintentionally pulling the trigger $(23.5 \%)$ was the most common associated circumstance, followed by mistakenly thinking the gun was unloaded $(9.0 \%)$ or mistakenly thinking the magazine was disengaged (6.0\%).

\section{Deaths of Undetermined Intent}

\section{Sex, Race/Ethnicity, and Age Group}

The 32 NVDRS states included in this report collected data on 4,436 incidents involving 4,470 deaths for which a determination of intent could not be made (Supplementary Table S1, https://stacks.cdc.gov/view/cdc/78990). The overall rate of deaths of undetermined intent was 2.4 per 100,000 population. The rate was higher among males than among females (3.2 and 1.6 per 100,000 population, respectively) (Supplementary Table S3, https://stacks.cdc.gov/view/ cdc/78990). Approximately $70 \%$ of persons for whom the manner of death was undetermined were aged 30-64 years. Rates were highest among adults aged $45-54$ years ( 4.0 per 100,000 population), followed by adults aged $30-34$ years (3.8 per 100,000 population), $25-29$ years (3.7 per 100,000 population), and 35-44 years (3.7 per 100,000 population). Non-Hispanic whites accounted for the majority (71.9\%) of deaths, whereas non-Hispanic American Indians/Alaska Natives had the highest rate (4.0 per 100,000 population). Among males, non-Hispanic blacks had the highest rate (5.0 per 100,000 population), followed by non-Hispanic American Indians/Alaska Natives (4.9 per 100,000 population).

\section{Method and Location of Injury}

Poisoning was the most common method of injury in deaths of undetermined intent (72.2\%) (Supplementary Table S3, https://stacks.cdc.gov/view/cdc/78990). No other method accounted for $>4 \%$ overall. The majority of deaths of undetermined intent occurred in a house/apartment (69.9\%), followed by a natural area $(4.6 \%)$, hotel/motel $(3.6 \%)$, and street/highway (3.4\%).

\section{Toxicology Results of Decedent}

Tests for antidepressants, benzodiazepines, and opioids were conducted for $40.1 \%, 44.6 \%$, and $77.4 \%$ of decedents, respectively (Supplementary Table S5, https://stacks.cdc. gov/view/cdc/78990). Results for antidepressants and benzodiazepines were positive in $54.2 \%$ and $49.9 \%$ of decedents tested for those substances, respectively. Results for opioids (including illicit or prescription drugs) were positive in $79.2 \%$ of decedents tested.

\section{Precipitating Circumstances}

Precipitating circumstances were known in $85.6 \%$ of deaths of undetermined intent (Supplementary Table S4, https:// stacks.cdc.gov/view/cdc/78990). Of those, a current diagnosed mental health problem was reported for $36.7 \%$ of decedents; depression/dysthymia (53.6\%), anxiety disorder (23.9\%), and bipolar disorder $(22.8 \%)$ were the most common diagnoses. 
Current depressed mood was reported for $9.9 \%$ of decedents, and $21.1 \%$ were receiving mental health treatment at the time of their death. Substance abuse problems (other than alcohol) $(66.2 \%)$ and alcohol problems (28.1\%) were the most commonly reported circumstances. Physical health problems (13.0\%) and a crisis during the preceding or upcoming 2 weeks (12.0\%) were other life stressors identified in deaths of undetermined intent. Among decedents, 10.1\% had a history of suicidal thoughts or plans, $8.3 \%$ had a history of suicide attempts, and $4.7 \%$ had disclosed intent to die by suicide.

\section{Suicides Among Youths Aged 10-24 Years}

\section{Sex, Race/Ethnicity, and Age Group}

The 32 NVDRS states included in this report collected data on 3,655 suicides among youths aged 10-24 years that occurred during 2016 (Table 10). Of these decedents, 911 (24.9\%) were aged $10-17$ years and 2,744 (75.1\%) were aged 18-24 years. The majority of youth decedents were male (78.7\%) and non-Hispanic white (68.1\%).

\section{Method and Location of Injury}

The most common method used was a firearm (44.7\%), followed by hanging/strangulation/suffocation (40.3\%) and poisoning (6.3\%) (Table 10). Hanging/strangulation/ suffocation was the method used in approximately half (49.3\%) of suicides among youths aged 10-17 years compared with $37.4 \%$ of suicides among youths aged $18-24$ years. The majority $(69.4 \%)$ of youth suicides occurred in a house/ apartment, followed by a natural area $(6.9 \%)$, a motor vehicle (5.0\%), a street/highway (2.8\%), and a park/playground/ sports or athletic area (2.5\%). However, a greater percentage of suicides among youths aged 10-17 years occurred at home than among youths aged 18-24 years $(80.9 \%$ and $65.6 \%$, respectively), and a greater percentage of suicides among those aged 18-24 years occurred in a motor vehicle compared with those aged $10-17$ years $(6.1 \%$ and $1.6 \%$, respectively).

\section{Toxicology Results of Decedent}

Tests for alcohol were conducted for approximately half of all suicide decedents aged 10-17 years and 18-24 years (50.8\% and 53.2\%, respectively) (Table 11). Among those tested, youths aged 18-24 years more commonly tested positive for alcohol compared with youths aged 10-17 years (39.0\% and $10.8 \%$, respectively); of those, $64.7 \%$ of youths aged 18-24 years had BACs $\geq 0.08 \mathrm{~g} / \mathrm{dL}$ compared with $36.0 \%$ of youths aged 10-17 years. Among youths tested for antidepressants, $28.3 \%$ of those aged $10-17$ years tested positive compared with $22.8 \%$ among those aged $18-24$ years. In contrast, youths aged 18-24 years tested positive for benzodiazepines, marijuana, and opioids more commonly than those aged $10-17$ years.

\section{Precipitating Circumstances}

Precipitating circumstances were known for $90.1 \%$ of suicide decedents aged 10-24 years (Table 12). Of those with known circumstance information, similar proportions of youths aged $10-17$ years and $18-24$ years had a current diagnosed mental health problem $(46.3 \%$ and $45.7 \%$, respectively) or were described as being depressed at the time of their death $35.6 \%$ and $36.3 \%$, respectively). Among youths aged 10-24 years with a diagnosed mental health problem, depression/dysthymia (70.9\%), anxiety disorder (17.6\%), and bipolar disorder (12.8\%) were the most common diagnoses. Attention deficit disorder/ attention deficit hyperactivity disorder was more commonly diagnosed among youths aged 10-17 years than among those aged $18-24$ years $(16.7 \%$ and $5.9 \%$, respectively), whereas bipolar disorder and schizophrenia were more commonly diagnosed among youths aged 18-24 years than among those aged $10-17$ years $(15.5 \%$ versus $4.8 \%$ and $6.5 \%$ versus $<1.0 \%$, respectively). A slightly greater percentage of youths aged 10-17 years was known to be receiving mental health treatment at the time of death than those aged 18-24 years (30.3\% and $24.3 \%$, respectively). Substance abuse problems and alcohol problems were reported more often among youths aged $18-24$ years than among those aged $10-17$ years $(21.2 \%$ versus $9.1 \%$ and $12.4 \%$ versus $3.2 \%$, respectively).

With respect to interpersonal relationships, intimate partner problems were a common precipitating circumstance among youths aged $10-24$ years $(31.0 \%)$; approximately one in three youths aged 18-24 years (32.9\%) and one in four youths aged 10-17 years $(25.1 \%)$ were known to have an intimate partner problem contribute to their death (Table 12). Family relationship problems were reported for $17.0 \%$ of suicides among youths aged 10-24 years; however, among interpersonal suicide circumstances, family relationship problems accounted for the largest percentage difference between youths aged $10-17$ years and youths aged $18-24$ years $(32.6 \%$ versus $11.9 \%$, respectively). A crisis during the previous or upcoming 2 weeks was the most frequently documented life stressor for youths aged $10-24$ years (33.4\%), followed by an argument or conflict $(22.4 \%)$, school problems $(10.0 \%)$, and a recent criminal legal problem (8.0\%). A crisis was more common among youths aged 10-17 years than among those aged $18-24$ years (38.9\% versus $31.5 \%$, respectively). Among life stressors, school problems accounted for the largest percentage difference between youths aged 10-17 years and youths aged $18-24$ years (26.0\% versus $4.7 \%$, respectively).

Among other circumstances related to the suicide event, $35.7 \%$ of youths aged $10-24$ years had a history of suicidal 
thoughts or plans, $34.6 \%$ left a suicide note, and $23.1 \%$ had a history of suicide attempts (Table 12). A greater percentage among youths aged 10-17 years left a suicide note than among those aged $18-24$ years (39.6\% versus 32.9\%, respectively). Approximately one fourth of decedents aged 10-24 years (25.9\%) disclosed suicidal intent to another person. Among youths aged 10-17 years who disclosed intent, the greatest proportion of disclosure was to a friend/colleague (32.3\%), followed by a family member $(28.4 \%)$ and a previous or current intimate partner (18.9\%). Among youths aged 18-24 years who disclosed intent, the greatest proportion of disclosure was to a previous or current intimate partner $(35.6 \%)$, followed by a family member $(29.8 \%)$ and a friend/colleague (18.4\%).

\section{Discussion}

Violent deaths affect males and females and persons of all ages, races, and ethnicities. NVDRS data help identify disparities in and characteristics of populations particularly affected by fatal violence. Violence also occurs in different forms that are linked to one another in important ways. NVDRS not only provides details on specific manners of violent death, but also has the capacity to identify cross-cutting risk factors for multiple forms of violence. These details increase knowledge about the circumstances associated with violence and can help public health authorities and their partners develop and guide data-informed, effective approaches to violence prevention.

The occurrence of violent death varies greatly across states (1). Whereas this report summarizes 2016 data from 32 U.S. states, in 2019 NVDRS expanded data collection to include all 50 states, the District of Columbia, and Puerto Rico, achieving a truly national NVDRS (10). With nationwide expansion, NVDRS will provide more comprehensive, accessible, and actionable violent death information and equip all states and communities with data for appropriate public health action at the local level. The 32 states that provided data for this report accounted for $58.9 \%$ of violent deaths and represented $58 \%$ of the U.S. population in $2016(1,4)$. The nationwide expansion will allow CDC to provide valuable information on the scope of violent deaths in the United States, provide data for development of evidence-based violence prevention efforts at the national level, and allow every U.S. state to have access to this data at state, local, and regional levels.

Violence is preventable, and reducing violent deaths in communities is possible with evidence-based approaches (11). CDC developed technical packages to assist communities and states in identifying violence prevention approaches that are based on the best available evidence (11). The five technical packages include strategies; approaches; and specific programs, practices, and policies with evidence of effects on risk for child abuse and neglect, IPV, youth violence, sexual violence, and suicide. Each package considers the multifaceted and interactive effects of individual, relationship, family, school, and community factors that can influence violence-related outcomes. The strategies and approaches are intended to work together across the different levels of social ecology in a multisector effort to prevent violence. Sectors instrumental to the implementation of these packages include education, government, social services, justice, housing, faith-based organizations, and the private sector (11).

The findings in this report demonstrate that demographic variations in the manner of death from violence-related injuries persist. Suicides comprised the majority (62.3\%) of violent deaths collected in NVDRS and occurred at higher rates among non-Hispanic American Indians/Alaska Natives, followed by non-Hispanic whites. Suicide rates were highest among males and adults aged 45-64 years. Infants experienced homicide rates of 7.0 per 100,000 population, a rate that was 3.3 times the rate among children aged $1-4$ years, 8.8 times the rate among children aged 5-9 years, and 11.7 times the rate among youths aged 10-14 years.

The high homicide rate for infants reinforces the need for prioritizing child abuse and neglect prevention and intervention strategies. Creating safe, stable, and nurturing relationships and environments is essential for the prevention of child abuse and neglect. CDC's child abuse and neglect prevention technical package (12) identified the following evidence-based strategies and approaches to help prevent child abuse and neglect: 1) strengthening economic supports for families, 2) changing social norms to support parents and positive parenting, 3) providing quality care and education early in life, 4) enhancing parenting skills to promote healthy child development, and 5) intervening to lessen harms and prevent future risk. Child abuse and neglect is preventable, and the specific approaches described in the technical package combined with creating safe, stable, and nurturing relationships and environments (13) can help reduce homicides of children and infants as well as child maltreatment and other adverse childhood experiences.

Homicide rates were highest among persons aged 20-29 years, especially males. For both males and females, rates were highest among non-Hispanic blacks and American Indians/Alaska Natives. Among males, the homicide rate among non-Hispanic blacks was 41.5 per 100,000 population, a rate 2.7 times that for non-Hispanic American Indian/Alaska Native and approximately 14 times that for non-Hispanic white males.

Racial and ethnic minorities experience inequitable rates of violent injury and homicide, and these inequities are pervasive and persistent (14). The racial/ethnic inequities 
in homicide rates identified in this report, particularly among youths and young adult males, warrant prioritizing inequities related to race/ethnicity in violence prevention. These inequities result from the disproportionate exposure of racial and ethnic minorities to systemic inequities such as residential segregation, concentrated disadvantage, stress from experiencing racism, limited access to the best educational and employment opportunities, and other conditions that increase risk for experiencing violence (14-16). Racial and ethnic minority youths often live in communities with concentrated poverty, stressed economies, residential instability and neighborhood disorganization, access to firearms and illegal drugs, and low community cohesion and informal controls $(15,16)$. All these conditions are associated with violence and violence-related injuries (15). Prevention efforts will achieve greater population-level reductions in violence through the reduction of systemic inequities and by targeting salient neighborhood and community-level contributors to violence (17). Evaluations of programs such as Baltimore's Safe Streets, Crime Prevention Through Environmental Design (CPTED), business improvement districts, and policies such as the earned income tax credit (18) have confirmed the value in employing these types of community-level strategies in reducing violence. Evidence also suggests that these strategies and other universal policies that focus on general community improvements can have substantial impact on decreasing the race/ethnicity gap in violence (15).

A firearm was the most common method used in suicides. Lethal means, such as firearms, provide limited opportunity for intervention and have high case-fatality rates (19). Creating protective environments by reducing access to lethal means among persons at risk can be an effective strategy to prevent suicide (19). Past suicidal behavior and mental illness are important risk factors for suicide as well (19). In this report, $32.6 \%$ of suicide decedents had a history of suicidal thoughts or plans and $23.5 \%$ had disclosed their suicide intent. These precipitants are well documented as important risk factors to target in suicide prevention $(19,20)$. Mental health problems are also still the most commonly identified circumstance and continue to be a key focus for prevention. Less than one third of suicide decedents were known to be receiving treatment at the time of death, pointing to a gap between those receiving treatment and those who would likely benefit from it.

Although treatment for mental health problems is an essential component of suicide prevention, previous research with NVDRS data has documented that more than half of suicide decedents did not have a known mental health condition (21). That study indicated that multiple factors contribute to suicide among those with and without known mental health conditions. Although mental health conditions are important, other potential opportunities exist for prevention. For example, findings in this report illustrate that intimate partner problems and recent crises were also frequent precipitants of suicide.

CDC's suicide prevention technical package (19) contains the following seven strategies for reducing suicide and suicidal behaviors: 1) strengthen economic supports, 2) strengthen access and delivery of suicide care, 3 ) create protective environments, 4) promote connectedness, 5) teach coping and problem-solving skills, 6) identify and support persons at risk, and 7) lessen harms and prevent future risk. Each strategy includes examples of specific approaches that states and communities can implement to advance the strategy. This report's findings indicate that several approaches, including social-emotional learning programs, treatment for persons at risk for suicide and treatment to prevent reattempts, and enhancing parenting skills and family relationships, will be important to include when developing suicide prevention programs. Suicide prevention efforts are best achieved if these approaches and strategies are used in combination and reinforce one another to reduce risk for suicide and have cross-cutting impact on other forms of violence (19). By using NVDRS data, suicide prevention experts can guide planning and implementation and track outcomes of suicide prevention strategies and approaches within their states and communities. The strategies in the technical package support the goals and objectives of the National Strategy for Suicide Prevention (NSSP) (22) and the National Action Alliance for Suicide Prevention's priority to strengthen community-based prevention (23).

NVDRS homicide circumstance data indicate that homicides among males were most often precipitated by an argument or conflict or during the commission of a crime (predominately assault/homicide). In contrast, $43.2 \%$ of homicides among females were related to IPV; a current or former spouse/ intimate partner was identified as the perpetrator in half of homicides of women with known perpetrators. These findings were consistent with another NVDRS report that highlighted the differential impact of IPV-related homicides among young and racial and ethnic minority women (24).

Efforts to reduce IPV among women include screening women of childbearing age for IPV and referring those who have a positive screen to intervention services (25) and providing counseling services for pregnant women experiencing IPV (26). IPV screening that is conducted in a culturally sensitive way is important to minimize threats to safety. Strategies and approaches across different levels of the social ecology to prevent IPV also include providing support to survivors; empowering bystanders; engaging men and boys as allies $(27,28)$; disrupting developmental pathways toward IPV; creating protective home, school, and workplace environments (27); and teaching youths 
about safe and healthy relationships before they begin dating $(27,29,30)$. Evidence-based, cross-cutting prevention efforts that incorporate changing social norms, including harmful gender norms that condone violence, and societal conditions that serve to maintain harmful norms and inequality across sex, race/ethnicity, and income groups are also effective approaches outlined in CDC's IPV prevention technical package (27).

Relationship problems are frequent precipitants of suicides and homicides, underscoring that many forms of violence are interconnected and might share the same root causes (31). Therefore, the impact of violence prevention and intervention efforts can be broadened by understanding shared risk and protective factors and addressing multiple, connected forms of violence (31). This report's findings also support the need to implement programs that develop social and emotional skills (e.g., problem solving, conflict resolution, and individual coping skills) and cultivate supportive relationships to protect against violent injuries and death. For example, Safe Dates, a school-based program designed to reduce dating violence among adolescents, has indicated promise for reducing longterm physical and sexual dating violence as well as peer violence victimization and weapon carrying $(27,29,30)$. Furthermore, primary prevention strategies designed to teach skills that reduce aggressive behavior toward others and improve social skills, emotional well-being, and self-esteem can be targeted toward preadolescents and early adolescents before violent behaviors and patterns begin (17). CDC's youth violence technical package emphasizes the preventive effects of skill development programs for youths and prevention approaches that address relationships and influence school and community environments (17).

Substance use is another frequent precipitant of suicide and interpersonal violent behavior. Toxicology results documented a high prevalence of alcohol, especially with BACs $\geq 0.08 \mathrm{~g} / \mathrm{dL}$, among suicide and homicide decedents tested for substance use. Alcohol use is a robust predictor of suicidal behavior (32), victimization (33), and interpersonal violence perpetration $(17,27)$. Intoxication can lead to disinhibition, enhance feelings of hopelessness and depression, and impair judgment that can lead to impulsive behaviors (21). Alcohol use can also reduce awareness and perception of surrounding risks, thus increasing one's vulnerability to being victimized (34). Most deaths of undetermined intent were poisonings. Opioids (illicit or prescription) were the most common substances detected in deaths of undetermined intent; among those tested, $79.2 \%$ had positive opioid results. Whether these deaths were the result of unintentional drug poisoning or suicide is unknown. Opioid overdose has been recognized as an epidemic (35). CDC published Guideline for Prescribing Opioids for Chronic Pain to help address the epidemic; support safer prescribing practices; and reduce opioid misuse, opioid use disorder, and overdose (36). Other important activities include expanding naloxone availability and access to medicationassisted treatment, enhancing public health and public safety partnerships, and maximizing the ability of health systems to link persons to treatment and harm-reduction services (37).

NVDRS also collects more complete information than other data sources on legal intervention deaths (38) and unintentional firearm deaths (39). Findings from 32 states indicate that the largest proportion of deaths due to legal intervention was among non-Hispanic white males; however, the rate among non-Hispanic black males was approximately three times that of their white male counterparts, a finding consistent with previous studies $(40,41)$. Further analyses are needed to increase knowledge about the magnitude and circumstances of these deaths, which is essential to developing appropriate prevention strategies and monitoring their effectiveness. NVDRS also has been recognized as a reliable source of data on unintentional firearm deaths (39) and for its capability to provide details about victims and shooters (42). Findings in this report indicate that more than half of unintentional firearm deaths were self-inflicted, but approximately $35.3 \%$ were known to be inflicted by another person. Most of these deaths occurred while playing with a gun, accidentally pulling the trigger, or while thinking the gun was unloaded, particularly among children (43), highlighting the importance of safe storage practices and education about safe handling of firearms.

NVDRS data permit examination of violent deaths involving specific populations. Findings in this report indicate that most suicide decedents aged 10-24 years were non-Hispanic white males aged 18-24 years. Approximately half of all youths aged 10-17 years died by hanging/strangulation/suffocation, whereas the proportion of suicides that were from firearmrelated injuries was greater among youths aged 18-24 years (46.2\%). Among youth suicide decedents aged 10-24 years, approximately half had a diagnosed mental health condition. Depression was the most common diagnosis among all youth suicide decedents. The prevalence of attention deficit disorder/attention deficit hyperactivity disorder was greater among youths aged 10-17 years compared with youths aged 18-24 years (16.7\% versus $5.9 \%$, respectively). The most common precipitating circumstances associated with suicide among youths aged 10-24 years were interpersonal problems and a crisis during the previous or upcoming 2 weeks. However, the nature of the relationship problems experienced by older and younger youths differed. Family relationship problems were more commonly a precipitant among youths aged 10-17 years and intimate partner problems were more common among youths aged 18-24 years, reflecting the 
importance of different relationships at different points in youth development.

Several approaches might be helpful in reducing suicide among youths. Programs that promote connectedness through safe, stable, and nurturing relationships (e.g., Sources of Strength) have been reported to increase perceptions of adult support for suicidal youths and acceptability of help-seeking behaviors among high school students (44). Social-emotional learning programs (e.g., Youth Aware of Mental Health Program) (45) and parenting skill and family relationship programs (e.g., Strengthening Families 10-14) (46) teach coping and problem-solving skills to youths and their families. Youth Aware of Mental Health Program teaches adolescents aged 14-16 years about the risk and protective factors associated with suicide and enhances their problem-solving skills for dealing with adverse life events, stress, school, and other problems (45). Strengthening Families 10-14 improves parents' skills for disciplining, managing emotions and conflict, and communicating with their children; promotes youths' interpersonal and problem-solving skills; and creates family activities to build cohesion and positive parent-child interactions (46). Finally, identifying persons at risk for suicide and delivering treatment and support for these individuals through gatekeeper training can have a positive impact on suicide and its associated risk factors (19). Gatekeepers are community members (e.g., teachers, coaches, and primary health care providers) trained to identify persons who might be at risk for suicide and to respond effectively by facilitating treatment seeking and support services (19). Counties that implemented gatekeeper training through the Garret Lee Smith Memorial Suicide Prevention Program were found to have significantly lower suicide rates among youths aged 10-24 years 1 year after the training implementation than counties that did not implement the program (47).

NVDRS data have been used by states to examine the circumstances surrounding youth suicides and develop suicide prevention programs (48-51). For example, because the unadjusted suicide rate in Utah for youths aged 10-17 years had more than doubled during 2011-2015, in 2017 the Utah Department of Public Health collaborated with CDC to conduct an epidemiologic investigation of suicides among that age group (48). To identify precipitating circumstances for these suicides, data were analyzed from the Utah Violent Death Reporting System (UTVDRS) for years 2015-2017 (48). Precipitating circumstances were found to include mental health problems, depressed mood, a history of suicidal ideation, a recent crises such as family and intimate partner problems, school problems, and suicide of a friend or family member. Narrative data from UTVDRS identified further details about the precipitating circumstances. For instance, family conflict resulting from or resulting in the restriction of technology use (e.g., mobile phones, tablets, gaming systems, or laptops) was a factor in approximately $13 \%$ of suicides in which circumstances were known. Results of the investigation point to target areas for preventing suicide and promoting mental health. Data describing youth suicide in Utah have been used to support suicide prevention efforts such as the creation of a student safety and crisis tip line, a study focused on prevention of firearm suicides, and a position at the Office of the Medical Examiner to study youth suicide deaths (49). Furthermore, the nuanced findings regarding technology restriction as a contributing factor in youth suicides stimulate areas for future research $(48,49)$.

NVDRS data have been integral in ongoing statewide surveillance on suicide among youths. In Rhode Island, data from the Rhode Island Violent Death Reporting System (RIVDRS) were analyzed to identify precipitating circumstances of youth suicide deaths in the state during 2004-2014 (50). RIVDRS data indicated that 127 youths aged $\leq 25$ years had died by suicide in Rhode Island during the 10-year period. Analyses provided information on injury location and weapon type, indicating that most youths died at home by hanging/strangulation/suffocation. RIVDRS data also indicated that the majority (74\%) of decedents with a mental health problem at the time of death had a diagnosis of depression and dysthymia. The results prompted the development of Rhode Island's Suicide Prevention Initiative (SPI) to address the link between suicide and depression. SPI is a referral system that links school crisis team members with emergency service clinicians who provide emergency mental health assessments to youths in elementary, middle, and high schools who are experiencing crises and suicidal ideation (50).

Data from NVDRS also have been useful for multisectoral collaborations targeted at understanding and preventing youth suicide in states. The Kansas Youth Suicide Prevention Task Force was convened by the Kansas Attorney General's office in 2018 to examine statewide efforts to prevent youth suicide and recommend changes in practice, policy, or law aimed at preventing youth suicide in Kansas (51). Data from the Kansas Violent Death Reporting System (KSVDRS) were presented at the inaugural meeting of the task force. The KSVDRS presentation encompassed an introduction to NVDRS and information on suicides among Kansas youths aged 10-17 years. KSVDRS staff continue to share information with the task force on the basis of their experience collecting data and reporting on violent death and suicide throughout the state.

NVDRS also is relevant to two national prevention initiatives: NSSP and Healthy People 2020 (22,52). NSSP is a comprehensive national agenda for suicide prevention (22). 
In particular, NVDRS is relevant to NSSP goals of increasing timeliness and usefulness of surveillance systems related to suicide prevention and evaluating outcomes and effectiveness of suicide prevention interventions. Healthy People 2020 includes objectives for reducing the number of suicides, homicides, and firearm-related deaths and increasing the number of states that link data on violent deaths from death certificates, law enforcement reports, and coroner/medical examiner reports at state and local levels (52). NVDRS data can be used to measure states' progress toward these goals by allowing for the examination of changing patterns in circumstances and risk profiles, which is not possible with other data sources.

\section{Limitations}

The findings provided in this report are subject to at least eight limitations. First, NVDRS data are available from a limited number of states and therefore are not nationally representative.

Second, the availability, completeness, and timeliness of data are dependent on partnerships among state Violent Death Reporting System programs and state health departments, vital statistics registrars' offices, coroners/medical examiners, and law enforcement personnel. Data sharing and communication among partners is particularly challenging when states have independent county coroner systems rather than a centralized coroner/medical examiner system, a large number of law enforcement jurisdictions, or both. NVDRS incident data might be limited or incomplete for areas in which these datasharing relationships are not developed fully.

Third, toxicology data are not collected consistently across all states or for all alcohol and drug categories. Toxicology testing is not conducted for all decedents, so the percentages of those with positive results for specific substances might be affected by selective testing patterns in coroner/medical examiner offices (52).

Fourth, abstractors are limited to the data included in the investigative reports they receive. Reports might not fully reflect all information known about an incident, particularly for homicides and legal intervention deaths, when data are less readily available until after a full investigation and adjudication are completed.

Fifth, case definitions present challenges when a single death is classified differently in different documents (e.g., unintentional in a law enforcement report, homicide in a coroner/medical examiner report, and undetermined on the death certificate). NVDRS abstractors reconcile these discrepancies using standard NVDRS case definitions and select a single manner of death on the basis of all source documents; the manner of death assigned must be consistent with the manner of death noted in at least one source document.

Sixth, variations in coding might occur depending on the abstractor's level of experience. For this reason, CDC provides extensive abstractor guidance and training and data validation checks, and states conduct blinded reabstractions of cases to test consistency and identify training needs.

Seventh, medical and mental health information (e.g., type of condition and whether the decedent was currently receiving treatment) often are not captured directly from medical records but from coroner/medical examiner reports and the decedent's family members and friends. Therefore, completeness and accuracy of this information is limited by the knowledge of the informant.

Finally, protective factor data (i.e., characteristics or circumstances that reduce the risk for violent death) are not collected by NVDRS because of the nature of death certificates, coroner/medical examiner reports, and law enforcement reports, which typically contain only circumstances associated with risk factors.

\section{Conclusion}

Public health surveillance is the foundation for public health practice (53). Monitoring prevalence and incidence of violence-related fatal injuries, defining priorities, and informing programmatic and violence prevention activities are essential parts of public health surveillance (54). In 2018, NVDRS received funding for nationwide expansion. As of 2019, all 50 states, Puerto Rico, and the District of Columbia participate in NVDRS, a move toward achieving the ultimate goal of providing nationally representative data by including all states, all U.S. territories, and the District of Columbia. This expansion will not only make violent death information available for every state to develop local prevention efforts, but will allow for the system's capacity to measure the need for and effects of violence prevention policies, programs, and practices at the national level.

\section{Acknowledgments}

Contributors to this report, including participating state Violent Death Reporting Systems; participating state agencies, including state health departments, vital registrars' offices, coroners'/medical examiners' offices, crime laboratories, and local and state law enforcement agencies; partner organizations, including the Safe States Alliance, American Public Health Association, American College of Preventive Medicine, International Association of Chiefs of Police, National Sherriff's Association, National Association of Medical Examiners, National Association for Public Health Statistics and Information Systems, National Violence Prevention Network, 
Council of State and Territorial Epidemiologists, and Association of State and Territorial Health Officials; federal agencies, including the U.S. Department of Justice, Bureau of Justice Statistics; the National Institute for Occupational Safety and Health and National Center for Health Statistics, CDC; other stakeholders, researchers, and foundations, including Harvard University Injury Control Research Center and the Joyce Foundation.

\section{Conflict of Interest}

No conflicts of interest were reported.

\section{References}

1. CDC. Web-based Injury Statistics Query and Reporting System (WISQARS). Atlanta, GA: CDC; 2018. https://www.cdc.gov/injury/ wisqars/index.html

2. Blair JM, Fowler KA, Jack SP, Crosby AE. The National Violent Death Reporting System: overview and future directions. Inj Prev 2016;22(Suppl 1):i6-11. https://doi.org/10.1136/injuryprev-2015-041819

3. Bonnie RJ, Fulco CE, Livermore CT, eds. Reducing the burden of injury: advancing prevention and treatment. Washington DC: National Academy Press; 1999. https://www.nap.edu/read/6321/chapter/1

4. US Census Bureau. QuickFacts: United States. Washington, DC: US Department of Commerce, US Census Bureau; 2018. https://www. census.gov/quickfacts

5. Krug EG, Mercy JA, Dahlberg LL, Zwi AB. The world report on violence and health. Lancet 2002;360:1083-8. https://doi.org/10.1016/ S0140-6736(02)11133-0

6. World Health Organization. International classification of diseases, tenth revision. Geneva, Switzerland: World Health Organization; 2007. https://www.who.int/classifications/icd/en/

7. CDC. National Violent Death Reporting System (NVDRS) coding manual version 5.2 [Online]. Atlanta, GA: US Department of Health and Human Services, CDC; 2018. https://www.cdc.gov/violenceprevention/ pdf/nvdrs/NVDRS-WebCodingManual.pdf

8. Murphy SL, Xu J, Kochanek KD. Deaths: final data for 2010. Natl Vital Stat Rep 2013;61:1-117.

9. CDC. US census populations with bridged race categories. Hyattsville, MD: US Department of Health and Human Services, CDC, National Center for Health Statistics; 2016. https://www.cdc.gov/nchs/nvss/ bridged_race.htm

10. Barber C, Azrael D, Hemenway D. A truly national National Violent Death Reporting System. Inj Prev 2013;19:225-6. https://doi. org/10.1136/injuryprev-2013-040817

11. CDC. Technical packages for violence prevention: using evidencebased strategies in youth violence prevention efforts. Atlanta, GA: US Department of Health and Human Services, CDC; 2018. https://www. cdc.gov/violenceprevention/pub/technical-packages.html

12. Forston B, Klevens J, Merrick M, et al. Preventing child abuse and neglect: a technical package for policy, norm, and programmatic activities. Atlanta, GA: US Department of Health and Human Services, CDC; 2016.

13. CDC. Essentials for childhood: steps to create safe, stable, nurturing relationships and environments. Atlanta, GA: US Department of Health and Human Services, CDC, National Center for Injury Prevention and Control; 2014. http://www.governor.wa.gov/sites/default/files/ documents/BRCCF_20160614_ReadingMaterials_essentials_for_ childhood_framework.pdf

14. Sheats KJ, Irving SM, Mercy JA, et al. Violence-related disparities experienced by black youth and young adults: opportunities for prevention. Am J Prev Med 2018;55:462-9. https://doi.org/10.1016/j. amepre.2018.05.017
15. Sampson RJ, Morenoff JD, Raudenbush S. Social anatomy of racial and ethnic disparities in violence. Am J Public Health 2005;95:224-32. https://doi.org/10.2105/AJPH.2004.037705

16. Zimmerman GM, Messner SF. Individual, family background, and contextual explanations of racial and ethnic disparities in youths' exposure to violence. Am J Public Health 2013;103:435-42. https:// doi.org/10.2105/AJPH.2012.300931

17. Massetti GM, David-Ferdon C. Preventing violence among high-risk youth and communities with economic, policy, and structural strategies. MMWR Suppl 2016;65(No. Suppl 1). https://doi.org/10.15585/mmwr. su6501a9

18. David-Ferdon C, Vivolo-Kantor AM, Dahlberg LL, Marshall KJ, Rainford N, Hall JE. A comprehensive technical package for the prevention of youth violence and associated risk behaviors. Atlanta, GA: US Department of Health and Human Services, CDC, National Center for Injury Prevention and Control; 2016.

19. Stone D, Holland K, Bartholow B, Crosby A, Davis S, Wilkins N. Preventing suicide: a technical package of policy, programs, and practices. Atlanta, GA: US Department of Health and Human Services, CDC, National Center for Injury Prevention and Control; 2017.

20. Institute of Medicine. Reducing suicide: a national imperative. Washington, DC: National Academy of Science; 2002.

21. Stone DM, Simon TR, Fowler KA, et al. Vital signs: trends in state suicide rates-United States, 1999-2016 and circumstances contributing to suicide-27 states, 2015. MMWR Morb Mortal Wkly Rep 2018;67:617-24. https://doi.org/10.15585/mmwr.mm6722a1

22. US Office of the Surgeon General; National Action Alliance for Suicide Prevention. 2012 national strategy for suicide prevention: goals and objectives for action. Washington, DC: US Department of Health and Human Services; 2012.

23. National Action Alliance for Suicide Prevention. Action Alliance priorities; 2017. Washington, DC: National Action Alliance for Suicide Prevention. https://theactionalliance.org

24. Petrosky E, Blair JM, Betz CJ, Fowler KA, Jack SPD, Lyons BH. Racial and ethnic differences in homicides of adult women and the role of intimate partner violence-United States, 2003-2014. MMWR Morb Mortal Wkly Rep 2017;66:741-6. https://doi.org/10.15585/mmwr. mm6628a1

25. US Preventive Services Task Force. Final recommendation statement: intimate partner violence and abuse of elderly and vulnerable adults: screening. Rockville, MD: US Preventive Services Task Force; 2013. https://www.uspreventiveservicestaskforce.org/Page/Document/ RecommendationStatementFinal/intimate-partner-violence-and-abuseof-elderly-and-vulnerable-adults-screening

26. Kiely M, El-Mohandes AA, El-Khorazaty MN, Blake SM, Gantz MG. An integrated intervention to reduce intimate partner violence in pregnancy: a randomized controlled trial. Obstet Gynecol 2010;115:273-83. Erratum in: Obstet Gynecol 2011;117:1232. https://doi.org/10.1097/ AOG.0b013e3181cbd482

27. Niolon PH, Kearns M, Dills J, et al. Preventing intimate partner violence across the lifespan: a technical package of programs, policies and practices. Atlanta, GA: US Department of Health and Human Services, CDC, National Center for Injury Prevention and Control; 2017. https:// www.cdc.gov/violenceprevention/pdf/ipv-technicalpackages.pdf

28. Miller E, Tancredi DJ, McCauley HL, et al. "Coaching boys into men": a cluster-randomized controlled trial of a dating violence prevention program. J Adolesc Health 2012;51:431-8. https://doi.org/10.1016/j. jadohealth.2012.01.018

29. Foshee VA, Bauman KE, Ennett ST, Linder GF, Benefield T, Suchindran C. Assessing the long-term effects of the Safe Dates program and a booster in preventing and reducing adolescent dating violence victimization and perpetration. Am J Public Health 2004;94:619-24. https://doi.org/10.2105/AJPH.94.4.619 
30. Foshee VA, Reyes LM, Agnew-Brune CB, et al. The effects of the evidence-based Safe Dates dating abuse prevention program on other youth violence outcomes. Prev Sci 2014;15:907-16. https://doi. org/10.1007/s11121-014-0472-4

31. Wilkins N, Tsao B, Hertz M, Davis R, Klevens J. Connecting the dots: an overview of the links among multiple forms of violence. Atlanta, GA: US Department of Health and Human Services, CDC, National Center for Injury Prevention and Control; Oakland, CA: Prevention Institute; 2014. https://www.cdc.gov/violenceprevention/pdf/connecting_the_dots-a.pdf

32. Poorolajal J, Haghtalab T, Farhadi M, Darvishi N. Substance use disorder and risk of suicidal ideation, suicide attempt and suicide death: a meta-analysis. J Public Health (Oxf) 2016;38:e282-91. https://doi. org/10.1093/pubmed/fdv148

33. Devries KM, Child JC, Bacchus LJ, et al. Intimate partner violence victimization and alcohol consumption in women: a systematic review and meta-analysis. Addiction 2014;109:379-91. https://doi. org/10.1111/add.12393

34. Fromme K, Katz E, D'Amico E. Effects of alcohol intoxication on the perceived consequences of risk taking. Exp Clin Psychopharmacol 1997;5:14-23. https://doi.org/10.1037/1064-1297.5.1.14

35. Rudd RA, Aleshire N, Zibbell JE, Gladden RM. Increases in drug and opioid overdose deaths-United States, 2000-2014. MMWR Morb Mortal Wkly Rep 2016;64:1378-82. https://doi.org/10.15585/mmwr. mm6450a3

36. Dowell D, Haegerich TM, Chou R. CDC guideline for prescribing opioids for chronic pain-United States, 2016. MMWR Recomm Rep 2016;65(No. RR-1). https://doi.org/10.15585/mmwr.rr6501e1

37. Scholl L, Seth P, Kariisa M, Wilson N, Baldwin G. Drug and opioidinvolved overdose deaths-United States, 2013-2017. MMWR Morb Mortal Wkly Rep 2018;67:1419-27. https://doi.org/10.15585/mmwr. mm675152e1

38. Barber C, Azrael D, Cohen A, et al. Homicides by police: comparing counts from the National Violent Death Reporting System, vital statistics, and supplementary homicide reports. Am J Public Health 2016;106:922-7. https://doi.org/10.2105/AJPH.2016.303074

39. Barber C, Hemenway D. Too many or too few unintentional firearm deaths in official U.S. mortality data? Accid Anal Prev 2011;43:724-31. https://doi.org/10.1016/j.aap.2010.10.018

40. DeGue S, Fowler KA, Calkins C. Deaths due to use of lethal force by law enforcement: findings from the National Violent Death Reporting System, 17 U.S. states, 2009-2012. Am J Prev Med 2016;51(Suppl 3):S173-87. https://doi.org/10.1016/j.amepre.2016.08.027

41. Krieger N, Kiang MV, Chen JT, Waterman PD. Trends in U.S. deaths due to legal intervention among black and white men, age 15-34 years, by county income level: 1960-2010. Harvard Public Health Review; 2015. http://harvardpublichealthreview.org/190

42. Hemenway D, Barber C, Miller M. Unintentional firearm deaths: a comparison of other-inflicted and self-inflicted shootings. Accid Anal Prev 2010;42:1184-8. https://doi.org/10.1016/j.aap.2010.01.008
43. Fowler KA, Dahlberg LL, Haileyesus T, Gutierrez C, Bacon S. Childhood firearm injuries in the United States. Pediatrics 2017;140:e20163486. https://doi.org/10.1542/peds.2016-3486

44. Wyman PA, Brown CH, LoMurray M, et al. An outcome evaluation of the Sources of Strength suicide prevention program delivered by adolescent peer leaders in high schools. Am J Public Health 2010;100:1653-61. https://doi.org/10.2105/AJPH.2009.190025

45. Wasserman D, Hoven CW, Wasserman C, et al. School-based suicide prevention programmes: the SEYLE cluster-randomised, controlled trial. Lancet 2015;385:1536-44. https://doi.org/10.1016/ S0140-6736(14)61213-7

46. Spoth RL, Guyll M, Day SX. Universal family-focused interventions in alcohol-use disorder prevention: cost-effectiveness and cost-benefit analyses of two interventions. J Stud Alcohol 2002;63:219-28. https:// doi.org/10.15288/jsa.2002.63.219

47. Walrath C, Garraza LG, Reid H, Goldston DB, McKeon R. Impact of the Garrett Lee Smith youth suicide prevention program on suicide mortality. Am J Public Health 2015;105:986-93. https://doi.org/10.2105/ AJPH.2014.302496

48. Annor FB, Zwald ML, Wilkinson A, et al. Characteristics of and precipitating circumstances surrounding suicide among persons aged 10-17 years-Utah, 2011-2015. MMWR Morb Mortal Wkly Rep 2018;67:329-32. https://doi.org/10.15585/mmwr.mm6711a4

49. Utah Department of Health. Utah health status update: CDC investigation shows youth suicides in Utah increasing. Special edition 4. Salt Lake City, UT: Utah Department of Health; 2017. https://ibis.health.utah.gov/ibisph-view/pdf/opha/publication/hsu/ SE04_SuicideEpiAid.pdf

50. Jiang Y, Pearlman DN, Hill J, Viner-Brown S. Surveillance of suicide and suicide attempts among Rhode Island youth using multiple data sources. R I Med J (2013) 2016;99:53-6.

51. Kansas Youth Suicide Prevention Task Force. Kansas Youth Suicide Prevention Task Force state report. Topeka, KS: Office of the Attorney General; December 2018. https://www.ag.ks.gov/docs/default-source/ reports/ysptf/2018-youth-suicide-prevention-task-force-report. pdf?sfvrsn=33bcd01a_4

52. HealthyPeople.gov [Internet]. Healthy people 2020. Washington, DC: Office of Disease Prevention and Health Promotion; 2013. https://www. healthypeople.gov/2020/topics-objectives

53. Karch D, Crosby A, Simon T. Toxicology testing and results for suicide victims-13 states, 2004. MMWR Morb Mortal Wkly Rep 2006;55:1245-8.

54. CDC. CDC public health surveillance strategy report: 2014-2018. Atlanta, GA: US Department of Health and Human Services, CDC; September 2018. https://www.cdc.gov/surveillance/improvingsurveillance/index.html 


\begin{tabular}{|c|c|c|c|}
\hline Manner of death & $\begin{array}{l}\text { Death } \leq 1 \text { year } \\
\text { after injury }\end{array}$ & $\begin{array}{l}\text { Death }>1 \text { year } \\
\text { after injury }\end{array}$ & $\begin{array}{l}\text { Death any time } \\
\text { after injury }\end{array}$ \\
\hline Intentional self-harm (suicide) & X60-X84 & Y87.0 & U03 (attributable to terrorism) \\
\hline Assault (homicide) & X85-X99, Y00-Y09 & Y87.1 & U01, U02 (attributable to terrorism) \\
\hline Event of undetermined intent & Y10-Y34 & Y87.2, Y89.9 & $\mathrm{N} / \mathrm{A}$ \\
\hline $\begin{array}{l}\text { Unintentional exposure to } \\
\text { inanimate mechanical forces } \\
\text { (firearms) }\end{array}$ & W32-W34 & Y86 & $\mathrm{N} / \mathrm{A}$ \\
\hline $\begin{array}{l}\text { Legal intervention } \\
\text { (excluding executions, Y35.5) }\end{array}$ & Y35.0-Y35.4, Y35.6, Y35.7 & Y89.0 & $\mathrm{N} / \mathrm{A}$ \\
\hline
\end{tabular}

BOX 2. Methods used to inflict injury — National Violent Death Reporting System, 32 states, 2016

- Firearm: method that uses a powder charge to fire a projectile from the weapon (excludes BB gun, pellet gun, and compressed air-powered or gas-powered gun)

- Hanging/strangulation/suffocation (e.g., hanging by the neck, manual strangulation, or plastic bag over the head)

- Poisoning (e.g., fatal ingestion of a street drug, pharmaceutical, carbon monoxide, gas, rat poison, or insecticide)

- Sharp instrument (e.g., knife, razor, machete, or pointed instrument)

- Blunt instrument (e.g., club, bat, rock, or brick)

- Fall: being pushed or jumping

- Motor vehicle (e.g., car, bus, motorcycle, or other transport vehicle)

- Personal weapons (e.g., hands, fists, or feet)

- Drowning: inhalation of liquid (e.g., in bathtub, lake, or other source of water/liquid)

- Fire/burns: inhalation of smoke or the direct effects of fire or chemical burns

- Intentional neglect: starvation, lack of adequate supervision, or withholding of health care

- Other (single method): any method other than those already listed (e.g., electrocution, exposure to environment/weather, or explosives)

- Unknown: method not reported or not known 


\section{Suicide/Undetermined Intent}

- Intimate partner problem: decedent was experiencing problems with a current or former intimate partner.

- Suicide of family member or friend: decedent was distraught over, or reacting to, the suicide of a family member or friend.

- Other death of family member or friend: decedent was distraught over, or reacting to, the recent nonsuicide death of a family member or friend.

- Physical health problem: decedent was experiencing physical health problems (e.g., a recent cancer diagnosis or chronic pain).

- Job problem: decedent was either experiencing a problem at work or having a problem with joblessness.

- Recent criminal legal problem: decedent was facing criminal legal problems (e.g., recent or impending arrest or upcoming criminal court date).

- Noncriminal legal problem: decedent was facing civil legal problems (e.g., a child custody or civil lawsuit).

- Financial problem: decedent was experiencing problems (e.g., bankruptcy, overwhelming debt, or foreclosure of a home or business).

- Eviction or loss of home: decedent was experiencing a recent or impending eviction or other loss of housing or the threat of eviction or loss of housing.

- School problem: decedent was experiencing a problem related to school (e.g., poor grades, bullying, social exclusion at school, or performance pressures).

- Traumatic anniversary: the incident occurred on or near the anniversary of a traumatic event in the decedent's life.

- Exposure to disaster: decedent was exposed to a disaster (e.g., earthquake or bombing).

- Left a suicide note: decedent left a note, e-mail message, video, or other communication indicating intent to die by suicide.

- Disclosed suicide intent: decedent had recently expressed suicidal feelings to another person with time for that person to intervene.

- Disclosed intent to whom: type of person (e.g., family member or current or former intimate partner) to whom the decedent recently disclosed suicidal thoughts/plans.

- History of suicidal thoughts or plans: decedent had previously expressed suicidal thoughts or plans.

- History of suicide attempt: decedent had previously attempted suicide before the fatal incident.

\section{Homicide/Legal Intervention}

- Jealousy (lovers' triangle): jealousy or distress over an intimate partner's relationship or suspected relationship with another person.

- Stalking: pattern of unwanted harassing or threatening tactics by either the decedent or suspect.

- Prostitution: prostitution or related activity that includes prostitutes, pimps, clients, or others involved in such activity.

- Drug involvement: drug dealing, drug trade, or illegal drug use.

- Brawl: mutual physical fight involving three or more persons.

- Mercy killing: decedent wished to die because of terminal or hopeless disease or condition, and documentation indicates that the decedent wanted to be killed.

- Victim was a bystander: decedent was not the intended target in the incident (e.g., pedestrian walking past a gang fight).

- Victim was a police officer on duty: decedent was a law enforcement officer killed in the line of duty.

- Victim was an intervener assisting a crime victim: decedent was attempting to assist a crime victim at the time of the incident (e.g., a child attempts to intervene and is killed while trying to assist a parent who is being assaulted).

- Victim used a weapon: decedent used a weapon to attack or defend during the course of the incident.

- Intimate partner violence-related: incident is related to conflict between current or former intimate partners; includes the death of an intimate partner or nonintimate partner (e.g., child, parent, friend, or law enforcement officer) killed in an incident that originated in a conflict between intimate partners.

- Hate crime: decedent was selected intentionally because of his or her actual or perceived gender, religion, sexual orientation, race/ethnicity, or disability.

- Mentally ill suspect: suspect's attack on decedent was believed to be the direct result of a mental illness.

- Drive-by shooting: suspect drove near the decedent and fired a weapon while driving. 
- Walk-by assault: decedent was killed by a targeted attack (e.g., ambush) where the suspect fled on foot.

- Random violence: decedent was killed in a random act of violence (i.e., an act in which the suspect is not concerned with who is being harmed, just that someone is being harmed).

- Gang-related: incident resulted from gang activity or gang rivalry; not used if the decedent was a gang member and the death did not appear to result from gang activity.

- Justifiable self-defense: decedent was killed by a law enforcement officer in the line of duty or by a civilian in legitimate self-defense or in defense of others.

\section{All Manners of Death (Except Unintentional Firearm)}

- Current depressed mood: decedent was perceived by self or others to be feeling depressed at the time of death.

- Current diagnosed mental health problem: decedent was identified as having a mental health disorder or syndrome listed in the Diagnostic and Statistical Manual of Mental Disorders, Fourth Edition (DSM-IV), with the exception of alcohol and other substance dependence (which are captured in separate variables).

- Type of mental health diagnosis: identifies the type of DSM-IV diagnosis reported for the decedent.

- Current mental health treatment: decedent was receiving mental health treatment as evidenced by a current prescription for a psychotropic medication, visit or visits to a mental health professional, or participation in a therapy group within the previous 2 months.

- History of ever being treated for mental health problem: decedent was identified as having ever received mental health treatment.

- Alcohol problem: decedent was perceived by self or others to have a problem with, or to be addicted to, alcohol.

- Substance abuse problem (excludes alcohol): decedent was perceived by self or others to have a problem with, or be addicted to, a substance other than alcohol.

- Other addiction: decedent was perceived by self or others to have an addiction other than to alcohol or other substance abuse (e.g., gambling or sex).

- Family relationship problem: decedent was experiencing problems with a family member (other than an intimate partner).

- Other relationship problem (nonintimate): decedent was experiencing problems with a friend or associate (other than an intimate partner or family member).

- History of child abuse/neglect: as a child, decedent had history of physical, sexual, or psychological abuse; physical (including medical or dental), emotional, or educational neglect; exposure to a violent environment; or inadequate supervision by a caretaker.

- Caretaker abuse/neglect led to death: decedent was experiencing physical, sexual, or psychological abuse; physical (including medical or dental), emotional, or educational neglect; exposure to a violent environment; or inadequate supervision by a caretaker that led to death.

- Perpetrator of interpersonal violence during previous month: decedent perpetrated interpersonal violence during the previous month.

- Victim of interpersonal violence during previous month: decedent was the target of interpersonal violence during the previous month.

- Physical fight (two persons, not a brawl): a physical fight between two persons that resulted in the death of the decedent, who was either involved in the fight, a bystander, or trying to stop the fight.

- Argument or conflict: a specific argument or disagreement led to the victim's death.

- Precipitated by another crime: incident occurred as the result of another serious crime.

- Nature of crime: identifies the specific type of other crime that occurred during the incident (e.g., robbery or drug trafficking).

- Crime in progress: another serious crime was in progress at the time of the incident.

- Terrorist attack: decedent was injured in a terrorist attack, leading to death.

- Crisis during previous or upcoming 2 weeks: current crisis or acute precipitating event or events that either occurred during the previous 2 weeks or was impending in the following 2 weeks (e.g., a trial for a criminal offense begins the following week). Crises typically are associated with specific circumstance variables (e.g., job problem was a crisis or financial problem was a crisis).

- Other crisis: a crisis related to a death but not captured by any of the standard circumstances. 


\section{Unintentional Firearm Death}

\section{Context of Injury}

- Hunting: death occurred any time after leaving home for a hunting trip and before returning home from a hunting trip.

- Target shooting: shooter was aiming for a target and unintentionally hit the decedent; can be at a shooting range or an informal backyard setting (e.g., teenagers shooting at signposts on a fence).

- Loading/unloading gun: gun discharged when the shooter was loading or unloading ammunition.

- Cleaning gun: shooter pulled trigger or gun discharged while cleaning, repairing, assembling, or disassembling gun.

- Showing gun to others: gun was being shown to another person when it discharged or the trigger was pulled.

- Playing with gun: shooter was playing with a gun when it discharged.

- Celebratory firing: shooter fired gun in celebratory manner (e.g., firing into the air at midnight on New Year's Eve).

- Other context of injury: shooting occurred during some context other than those already described.

Mechanism of Injury

- Unintentionally pulled trigger: shooter unintentionally pulled the trigger (e.g., while grabbing the gun or holding it too tightly).

- Thought gun safety was engaged: shooter thought the safety was on and gun would not discharge.

- Thought unloaded/magazine disengaged: shooter thought the gun was unloaded because the magazine was disengaged.

- Thought gun was unloaded: shooter thought the gun was unloaded for other unspecified reason.

- Bullet ricocheted: bullet ricocheted from its intended target and struck the decedent.

- Gun fired due to defect or malfunction: gun had a defect or malfunctioned as determined by a trained firearm examiner.

- Gun fired while holstering: gun was being replaced or removed from holster or clothing.

- Gun was dropped: gun discharged when it was dropped.

- Gun fired while operating safety/lock: shooter unintentionally fired the gun while operating the safety/lock.

- Gun was mistaken for toy: gun was mistaken for a toy and was fired without the user understanding the danger.

- Other mechanism of injury: shooting occurred as the result of a mechanism not already described 
TABLE 1. Number, percentage,* and rate ${ }^{\dagger}$ of suicides among persons aged $\geq 10$ years, $\$$ by decedent's sex, age group, race/ethnicity, method used, and location in which injury occurred - National Violent Death Reporting System, 32 states, ${ }^{\text {I }} 2016$

\begin{tabular}{|c|c|c|c|c|c|c|}
\hline \multirow[b]{2}{*}{ Characteristic } & \multicolumn{2}{|c|}{ Male } & \multicolumn{2}{|c|}{ Female } & \multicolumn{2}{|c|}{ Total } \\
\hline & No. (\%) & Rate & No. (\%) & Rate & No. (\%) & Rate \\
\hline \multicolumn{7}{|l|}{ Age group (yrs) } \\
\hline $10-14$ & $168(<1.0)$ & 2.8 & $90(1.5)$ & 1.5 & $258(<1.0)$ & 2.2 \\
\hline $15-19$ & 927 (4.6) & 14.8 & $291(4.9)$ & 4.8 & $1,218(4.7)$ & 9.9 \\
\hline $20-24$ & $1,781(8.9)$ & 26.8 & $398(6.8)$ & 6.3 & $2,179(8.4)$ & 16.8 \\
\hline $25-29$ & $1,760(8.8)$ & 26.6 & $393(6.7)$ & 6.1 & $2,153(8.3)$ & 16.5 \\
\hline $30-34$ & $1,595(8.0)$ & 25.4 & $470(8.0)$ & 7.5 & $2,065(8.0)$ & 16.5 \\
\hline $35-44$ & $3,051(15.3)$ & 26.4 & $1,006(17.1)$ & 8.6 & $4,057(15.7)$ & 17.4 \\
\hline $45-54$ & $3,624(18.2)$ & 29.3 & $1,326(22.5)$ & 10.4 & $4,950(19.1)$ & 19.7 \\
\hline $55-64$ & $3,378(16.9)$ & 28.5 & $1,125(19.1)$ & 8.8 & $4,503(17.4)$ & 18.3 \\
\hline $65-74$ & $1,913(9.6)$ & 24.5 & $529(9.0)$ & 5.9 & $2,442(9.4)$ & 14.6 \\
\hline $75-84$ & $1,190(6.0)$ & 33.6 & $190(3.2)$ & 4.1 & $1,380(5.3)$ & 16.8 \\
\hline$\geq 85$ & $573(2.9)$ & 44.5 & $71(1.2)$ & 2.8 & $644(2.5)$ & 17.0 \\
\hline Unknown & $1(<1.0)$ & —** & $0(0.0)$ & - & $1(<1.0)$ & - \\
\hline \multicolumn{7}{|l|}{ Race/Ethnicity } \\
\hline White, non-Hispanic & $16,542(82.9)$ & 29.7 & $4,867(82.6)$ & 8.4 & $21,409(82.8)$ & 18.8 \\
\hline Black, non-Hispanic & $1,278(6.4)$ & 12.5 & $340(5.8)$ & 3.0 & $1,618(6.3)$ & 7.5 \\
\hline American Indian/Alaska Native, non-Hispanic & $331(1.7)$ & 42.8 & $123(2.1)$ & 15.1 & $454(1.8)$ & 28.6 \\
\hline Asian/Pacific Islander & $522(2.6)$ & 12.1 & $220(3.7)$ & 4.6 & $742(2.9)$ & 8.2 \\
\hline Hispanic ${ }^{\dagger \dagger}$ & $1,232(6.2)$ & 13.2 & $311(5.3)$ & 3.4 & $1,543(6.0)$ & 8.4 \\
\hline Other & $56(<1.0)$ & - & $28(<1.0)$ & - & $84(<1.0)$ & - \\
\hline Unknown & $0(0.0)$ & - & $0(0.0)$ & - & $0(0.0)$ & - \\
\hline \multicolumn{7}{|l|}{ Method } \\
\hline Firearm & $11,046(55.3)$ & 13.7 & $1,714(29.1)$ & 2.0 & $12,760(49.4)$ & 7.8 \\
\hline Hanging/strangulation/suffocation & $5,534(27.7)$ & 6.9 & $1,660(28.2)$ & 2.0 & $7,194(27.8)$ & 4.4 \\
\hline Poisoning & $1,795(9.0)$ & 2.2 & $1,940(32.9)$ & 2.3 & $3,735(14.4)$ & 2.3 \\
\hline Fall & $497(2.5)$ & 0.6 & $185(3.1)$ & 0.2 & $682(2.6)$ & 0.4 \\
\hline Sharp instrument & $437(2.2)$ & 0.5 & $92(1.6)$ & 0.1 & $529(2.0)$ & 0.3 \\
\hline $\begin{array}{l}\text { Motor vehicle (e.g., car, bus, motorcycle, other } \\
\text { transport vehicle) }\end{array}$ & $329(1.6)$ & 0.4 & $139(2.4)$ & 0.2 & $468(1.8)$ & 0.3 \\
\hline Drowning & $153(<1.0)$ & 0.2 & $106(1.8)$ & 0.1 & $259(1.0)$ & 0.2 \\
\hline Fire/burns & $75(<1.0)$ & 0.1 & $26(<1.0)$ & $<0.1$ & $101(<1.0)$ & 0.1 \\
\hline Blunt instrument & $6(<1.0)$ & - & $1(<1.0)$ & - & $7(<1.0)$ & - \\
\hline Personal weapons (e.g., hands, feet, fists) & $1(<1.0)$ & - & $1(<1.0)$ & - & $2(<1.0)$ & - \\
\hline Intentional neglect & $0(0.0)$ & - & $1(<1.0)$ & - & $1(<1.0)$ & - \\
\hline Other (single method) & $41(<1.0)$ & - & $11(<1.0)$ & - & $52(<1.0)$ & - \\
\hline Unknown & $47(<1.0)$ & - & $13(<1.0)$ & - & $60(<1.0)$ & - \\
\hline \multicolumn{7}{|l|}{ Location } \\
\hline House/apartment & $14,423(72.3)$ & 18.0 & $4,702(79.8)$ & 5.6 & $19,125(74.0)$ & 11.6 \\
\hline Natural area & $1,126(5.6)$ & 1.4 & $192(3.3)$ & 0.2 & $1,318(5.1)$ & 0.8 \\
\hline Motor vehicle & $1,054(5.3)$ & 1.3 & $229(3.9)$ & 0.3 & $1,283(5.0)$ & 0.8 \\
\hline Street/highway & $492(2.5)$ & 0.6 & $103(1.7)$ & 0.1 & $595(2.3)$ & 0.4 \\
\hline Hotel/motel & $433(2.2)$ & 0.5 & $140(2.4)$ & 0.2 & $573(2.2)$ & 0.3 \\
\hline Parking lot/public garage/public transport & $353(1.8)$ & 0.4 & $74(1.3)$ & 0.1 & $427(1.7)$ & 0.3 \\
\hline Other location $\S \S$ & $1,878(9.4)$ & - & $372(6.3)$ & - & $2,250(8.7)$ & - \\
\hline Unknown & $202(1.0)$ & - & 77 (1.3) & - & $279(1.1)$ & - \\
\hline Total & $19,961(100)$ & 24.8 & $5,889(100)$ & 7.0 & $25,850(100)$ & 15.7 \\
\hline
\end{tabular}

* Percentages might not total $100 \%$ due to rounding.

+ Per 100,000 population.

$\S$ Suicide is not reported for decedents aged $<10$ years, as per standard in the suicide prevention literature. Denominators for the suicide rates represent the total population aged $\geq 10$ years.

I Alaska, Arizona, Colorado, Connecticut, Georgia, Hawaii, Illinois, Indiana, lowa, Kansas, Kentucky, Maine, Maryland, Massachusetts, Michigan, Minnesota, New Hampshire, New Jersey, New Mexico, New York, North Carolina, Ohio, Oklahoma, Oregon, Pennsylvania, Rhode Island, South Carolina, Utah, Vermont, Virginia, Washington, and Wisconsin. Illinois, Pennsylvania, and Washington collected data on $\geq 80 \%$ of violent deaths in the state, in accordance with requirements under which the state was funded. Denominators for the rates for these three states represent only the populations of the counties from which the data were collected.

** Rates are not reported when number of decedents is $<20$ or when characteristic response is "other" or "unknown."

t+ Includes persons of any race.

$\S \S$ Other location includes (in descending order): park/playground/sports or athletic area, jail/prison, railroad tracks, bridge, commercial/retail area, farm, hospital or medical facility, supervised residential facility, cemetery/graveyard/other burial ground, preschool/school/college/school bus, industrial or construction area, office building, abandoned house/building/warehouse, synagogue/church/temple, bar/nightclub, and other unspecified location. 
TABLE 2. Number* and percentage of suicide decedents who were tested for alcohol and drugs whose results were positive, ${ }^{\dagger}$ by toxicology variables - National Violent Death Reporting System, 32 states, $\$ 2016$

\begin{tabular}{lcrrr}
\hline & \multicolumn{1}{c}{ Tested } & & Positive \\
\cline { 2 - 2 } Toxicology variable & No. $(\%)$ & & No. $(\%)$ \\
\hline BAC & $13,310(51.5)$ & & $5,350(40.2)$ \\
Alcohol $<0.08 \mathrm{~g} / \mathrm{dL}$ & & & $1,582(29.6)$ \\
Alcohol $\geq 0.08 \mathrm{~g} / \mathrm{dL}$ & & & $3,448(64.4)$ \\
Alcohol positive, level unknown & & & $320(6.0)$ \\
Amphetamines & & & & \\
Anticonvulsants & $5,577(36.5)$ & & $1,063(11.3)$ \\
Antidepressants & $7,159(27.6)$ & & $945(17.0)$ \\
Antipsychotics & $5,760(22.3)$ & & $2,871(40.1)$ \\
Barbiturates & $7,915(30.6)$ & & $207(10.2)$ \\
Benzodiazepines & $10,070(39.0)$ & & $3,107(30.9)$ \\
Carbon monoxide & $2,002(7.7)$ & & $615(30.7)$ \\
Cocaine & $9,738(37.7)$ & & $654(6.7)$ \\
Marijuana & $8,269(32.0)$ & & $1,916(23.2)$ \\
Muscle relaxants & $5,836(22.6)$ & & $435(7.5)$ \\
Opioids & $10,532(40.7)$ & & $2,896(27.5)$ \\
Other drugs/substances $* *$ & $6,005(23.2)$ & & $4,722(78.6)$ \\
\hline
\end{tabular}

Abbreviation: $\mathrm{BAC}=$ blood alcohol concentration.

${ }^{*} \mathrm{~N}=25,850$

† Percentage is of decedents tested for toxicology variable.

$\S$ Alaska, Arizona, Colorado, Connecticut, Georgia, Hawaii, Illinois, Indiana, lowa, Kansas, Kentucky, Maine, Maryland, Massachusetts, Michigan, Minnesota, New Hampshire, New Jersey, New Mexico, New York, North Carolina, Ohio, Oklahoma, Oregon, Pennsylvania, Rhode Island, South Carolina, Utah, Vermont, Virginia, Washington, and Wisconsin. Illinois, Pennsylvania, and Washington collected data on $\geq 80 \%$ of violent deaths in the state, in accordance with requirements under which the state was funded.

" $B A C \geq 0.08 \mathrm{~g} / \mathrm{dL}$ is over the legal limit for impaired driving.

** Other drugs/substances indicated if any results were positive; levels for these drugs/substances are not measured. 
TABLE 3. Number* and percentage ${ }^{\dagger}$ of suicides among persons aged $\geq 10$ years, $\$$ by precipitating circumstances and decedent's sex - National Violent Death Reporting System, 32 states, " 2016

\begin{tabular}{|c|c|c|c|}
\hline & Male & Female & Total \\
\hline Precipitating circumstance & No. (\%) & No. (\%) & No. (\%) \\
\hline \multicolumn{4}{|l|}{ Mental health/Substance abuse } \\
\hline Current diagnosed mental health problem ${ }^{* *}$ & $8,044(44.3)$ & $3,533(64.3)$ & $11,577(49.0)$ \\
\hline Depression/dysthymia & $5,900(73.3)$ & $2,665(75.4)$ & $8,565(74.0)$ \\
\hline Anxiety disorder & $1,327(16.5)$ & $802(22.7)$ & $2,129(18.4)$ \\
\hline Bipolar disorder & $980(12.2)$ & $665(18.8)$ & $1,645(14.2)$ \\
\hline Schizophrenia & $455(5.7)$ & $169(4.8)$ & $624(5.4)$ \\
\hline PTSD & $461(5.7)$ & $129(3.7)$ & $590(5.1)$ \\
\hline ADD/ADHD & $211(2.6)$ & $55(1.6)$ & $266(2.3)$ \\
\hline OCD & $48(<1.0)$ & $10(<1.0)$ & $58(<1.0)$ \\
\hline Eating disorder & $1(<1.0)$ & $23(<1.0)$ & $24(<1.0)$ \\
\hline Other & $534(6.6)$ & $184(5.2)$ & $718(6.2)$ \\
\hline Unknown & $704(8.8)$ & $314(8.9)$ & $1,018(8.8)$ \\
\hline Current depressed mood & $6,745(37.2)$ & $1,983(36.1)$ & $8,728(36.9)$ \\
\hline History of ever being treated for a mental health problem & $5,886(32.5)$ & $2,803(51.0)$ & $8,689(36.8)$ \\
\hline Current mental health treatment & $4,180(23.0)$ & $2,209(40.2)$ & $6,389(27.0)$ \\
\hline Alcohol problem & $3,360(18.5)$ & $838(15.3)$ & $4,198(17.8)$ \\
\hline Substance abuse problem (excludes alcohol) & $2,930(16.2)$ & $1,030(18.8)$ & $3,960(16.8)$ \\
\hline Other addiction (e.g., gambling, sex) & $170(<1.0)$ & $24(<1.0)$ & $194(<1.0)$ \\
\hline \multicolumn{4}{|l|}{ Interpersonal } \\
\hline Intimate partner problem & $5,253(29.0)$ & $1,391(25.3)$ & $6,644(28.1)$ \\
\hline Family relationship problem & $1,576(8.7)$ & $667(12.1)$ & $2,243(9.5)$ \\
\hline Other death of family member or friend & $1,074(5.9)$ & $391(7.1)$ & $1,465(6.2)$ \\
\hline Suicide of family member or friend & $407(2.2)$ & $153(2.8)$ & $560(2.4)$ \\
\hline Perpetrator of interpersonal violence during past month & $511(2.8)$ & $47(<1.0)$ & $558(2.4)$ \\
\hline Other relationship problem (nonintimate) & $390(2.2)$ & $133(2.4)$ & $523(2.2)$ \\
\hline Victim of interpersonal violence during past month & $44(<1.0)$ & $53(<1.0)$ & $97(<1.0)$ \\
\hline \multicolumn{4}{|l|}{ Life stressor } \\
\hline Crisis during previous or upcoming 2 weeks & $5,853(32.3)$ & $1,527(27.8)$ & $7,380(31.2)$ \\
\hline Physical health problem & $4,028(22.2)$ & $1,236(22.5)$ & $5,264(22.3)$ \\
\hline Argument or conflict & $2,926(16.1)$ & $909(16.6)$ & $3,835(16.2)$ \\
\hline Job problem & $2,034(11.2)$ & $376(6.8)$ & $2,410(10.2)$ \\
\hline Financial problem & $1,670(9.2)$ & $382(7.0)$ & $2,052(8.7)$ \\
\hline Recent criminal legal problem & $1,771(9.8)$ & $248(4.5)$ & $2,019(8.5)$ \\
\hline Eviction or loss of home & $718(4.0)$ & $208(3.8)$ & 926 (3.9) \\
\hline Noncriminal legal problem & $635(3.5)$ & $163(3.0)$ & 798 (3.4) \\
\hline School problem & $288(1.6)$ & $93(1.7)$ & $381(1.6)$ \\
\hline History of child abuse/neglect & $142(<1.0)$ & $107(1.9)$ & $249(1.1)$ \\
\hline Physical fight (two persons, not a brawl) & $203(1.1)$ & $30(<1.0)$ & $233(<1.0)$ \\
\hline Traumatic anniversary & $113(<1.0)$ & $45(<1.0)$ & $158(<1.0)$ \\
\hline Caretaker abuse/neglect led to death & $10(<1.0)$ & $12(<1.0)$ & $22(<1.0)$ \\
\hline Exposure to disaster & $22(<1.0)$ & $0(0.0)$ & $22(<1.0)$ \\
\hline \multicolumn{4}{|l|}{ Crime and criminal activity } \\
\hline Precipitated by another crime & $728(4.0)$ & $60(1.1)$ & $788(3.3)$ \\
\hline Crime in progress ${ }^{\dagger \dagger}$ & $237(32.6)$ & $16(26.7)$ & $253(32.1)$ \\
\hline Terrorist attack & $0(0.0)$ & $0(0.0)$ & $0(0.0)$ \\
\hline \multicolumn{4}{|l|}{ Suicide event } \\
\hline Left a suicide note & $5,776(31.8)$ & $2,164(39.4)$ & $7,940(33.6)$ \\
\hline History of suicidal thoughts or plans & $5,733(31.6)$ & $1,972(35.9)$ & $7,705(32.6)$ \\
\hline History of suicide attempt & $2,990(16.5)$ & $1,809(32.9)$ & $4,799(20.3)$ \\
\hline \multicolumn{4}{|l|}{ Suicide disclosure } \\
\hline Disclosed suicide intent & $4,320(23.8)$ & $1,234(22.5)$ & $5,554(23.5)$ \\
\hline
\end{tabular}

See table footnotes on next page. 
TABLE 3. (Continued) Number* and percentage ${ }^{\dagger}$ of suicides among persons aged $\geq 10$ years, ${ }^{\S}$ by precipitating circumstances and decedent's sex — National Violent Death Reporting System, 32 states, 2016

\begin{tabular}{|c|c|c|c|}
\hline & Male & Female & Total \\
\hline Precipitating circumstance & No. (\%) & No. (\%) & No. (\%) \\
\hline \multicolumn{4}{|l|}{ Disclosed intent to whom $\$ \S$} \\
\hline Previous or current intimate partner & $1,659(38.4)$ & $440(35.7)$ & $2,099(37.8)$ \\
\hline Other family member & $1,279(29.6)$ & $358(29.0)$ & $1,637(29.5)$ \\
\hline Friend/colleague & $562(13.0)$ & $183(14.8)$ & $745(13.4)$ \\
\hline Health care worker & $177(4.1)$ & $72(5.8)$ & $249(4.5)$ \\
\hline Neighbor & $40(<1.0)$ & $14(1.1)$ & $54(<1.0)$ \\
\hline Other person & $342(7.9)$ & $69(5.6)$ & $411(7.4)$ \\
\hline Unknown & $261(6.0)$ & $98(7.9)$ & $359(6.5)$ \\
\hline Total ११ & $18,138(90.9)$ & $5,492(93.3)$ & $23,630(91.4)$ \\
\hline
\end{tabular}

Abbreviations: $\mathrm{ADD} / \mathrm{ADHD}=$ attention deficit disorder/attention deficit hyperactivity disorder; $\mathrm{OCD}=$ obsessive-compulsive disorder; $\mathrm{PTSD}=$ posttraumatic stress disorder.

* Includes suicides with one or more precipitating circumstances. More than one circumstance could have been present per decedent.

t Denominator includes those suicides with one or more precipitating circumstances. The sum of percentages in columns exceeds $100 \%$ because more than one circumstance could have been present per decedent.

$\S$ Suicide is not reported for decedents aged $<10$ years, as per standard in the suicide prevention literature. Denominators for the suicide rates represent the total population aged $\geq 10$ years.

ף Alaska, Arizona, Colorado, Connecticut, Georgia, Hawaii, Illinois, Indiana, lowa, Kansas, Kentucky, Maine, Maryland, Massachusetts, Michigan, Minnesota, New Hampshire, New Jersey, New Mexico, New York, North Carolina, Ohio, Oklahoma, Oregon, Pennsylvania, Rhode Island, South Carolina, Utah, Vermont, Virginia, Washington, and Wisconsin. Illinois, Pennsylvania, and Washington collected data on $\geq 80 \%$ of violent deaths in the state, in accordance with requirements under which the state was funded. Denominators for the rates for these three states represent only the populations of the counties from which the data were collected.

** Includes decedents with one or more diagnosed current mental health problems; therefore, sums of percentages for the diagnosed conditions exceed $100 \%$. Denominator includes the number of decedents with one or more current diagnosed mental health problems.

t+ Denominator includes those decedents involved in an incident that was precipitated by another crime.

$\S \S$ Denominator includes decedents who disclosed intent.

१ศ Circumstances were unknown for 2,220 decedents (1,823 males and 397 females); total no. of suicide decedents $=25,850$ (19,961 males and 5,889 females). 
TABLE 4. Number, percentage, ${ }^{*}$ and rate $^{\dagger}$ of homicides, by decedent's sex, age group, race/ethnicity, method used, location in which injury occurred, and victim-suspect relationship — National Violent Death Reporting System, 32 states, $\$ 2016$

\begin{tabular}{|c|c|c|c|c|c|c|}
\hline \multirow[b]{2}{*}{ Characteristic } & \multicolumn{2}{|c|}{ Male } & \multicolumn{2}{|c|}{ Female } & \multicolumn{2}{|c|}{ Total } \\
\hline & No. (\%) & Rate & No. (\%) & Rate & No. (\%) & Rate \\
\hline \multicolumn{7}{|l|}{ Age group (yrs) } \\
\hline$<1$ & $80(<1.0)$ & 6.9 & $77(3.6)$ & 7.0 & $157(1.5)$ & 7.0 \\
\hline $1-4$ & 99 (1.2) & 2.1 & $92(4.3)$ & 2.1 & $191(1.8)$ & 2.1 \\
\hline $5-9$ & $52(<1.0)$ & 0.9 & $37(1.7)$ & 0.6 & $89(<1.0)$ & 0.8 \\
\hline $10-14$ & $42(<1.0)$ & 0.7 & $35(1.6)$ & 0.6 & $77(<1.0)$ & 0.6 \\
\hline $15-19$ & $841(10.3)$ & 13.4 & $152(7.1)$ & 2.5 & $993(9.6)$ & 8.1 \\
\hline $20-24$ & $1,614(19.7)$ & 24.3 & $229(10.7)$ & 3.6 & $1,844(17.8)$ & 14.2 \\
\hline $25-29$ & $1,378(16.8)$ & 20.8 & $241(11.2)$ & 3.7 & $1,619(15.7)$ & 12.4 \\
\hline $30-34$ & $1,056(12.9)$ & 16.8 & $208(9.7)$ & 3.3 & $1,264(12.2)$ & 10.1 \\
\hline $35-44$ & $1,359(16.6)$ & 11.7 & $354(16.5)$ & 3.0 & $1,713(16.6)$ & 7.3 \\
\hline $45-54$ & $828(10.1)$ & 6.7 & $289(13.4)$ & 2.3 & $1,117(10.8)$ & 4.4 \\
\hline $55-64$ & $498(6.1)$ & 4.2 & $217(10.1)$ & 1.7 & $715(6.9)$ & 2.9 \\
\hline $65-74$ & $228(2.8)$ & 2.9 & $108(5.0)$ & 1.2 & $336(3.3)$ & 2.0 \\
\hline $75-84$ & $88(1.1)$ & 2.5 & $65(3.0)$ & 1.4 & $153(1.5)$ & 1.9 \\
\hline$\geq 85$ & $20(<1.0)$ & 1.6 & $42(2.0)$ & 1.7 & $62(<1.0)$ & 1.6 \\
\hline Unknown & $3(<1.0)$ & - & $3(<1.0)$ & - & $6(<1.0)$ & - \\
\hline \multicolumn{7}{|l|}{ Race/Ethnicity } \\
\hline White, non-Hispanic & $1,892(23.1)$ & 3.0 & $1,040(48.4)$ & 1.6 & $2,932(28.4)$ & 2.3 \\
\hline Black, non-Hispanic & $5,010(61.2)$ & 41.5 & $781(36.3)$ & 5.9 & $5,792(56.0)$ & 22.9 \\
\hline American Indian/Alaska Native, non-Hispanic & $141(1.7)$ & 15.4 & $46(2.1)$ & 4.8 & $187(1.8)$ & 10.0 \\
\hline Asian/Pacific Islander & $104(1.3)$ & 2.1 & $60(2.8)$ & 1.1 & $164(1.6)$ & 1.6 \\
\hline Hispanic ${ }^{* *}$ & $998(12.2)$ & 8.6 & $214(10.0)$ & 1.9 & $1,212(11.7)$ & 5.3 \\
\hline Other & $40(<1.0)$ & - & $8(<1.0)$ & - & $48(<1.0)$ & - \\
\hline Unknown & $1(<1.0)$ & - & $0(0.0)$ & - & $1(<1.0)$ & - \\
\hline \multicolumn{7}{|l|}{ Method } \\
\hline Firearm & $6,453(78.8)$ & 7.0 & $1,170(54.4)$ & 1.2 & $7,623(73.8)$ & 4.1 \\
\hline Sharp instrument & $761(9.3)$ & 0.8 & $337(15.7)$ & 0.4 & $1,098(10.6)$ & 0.6 \\
\hline Personal weapons (e.g., hands, feet, fists) & $290(3.5)$ & 0.3 & $133(6.2)$ & 0.1 & $423(4.1)$ & 0.2 \\
\hline Blunt instrument & $271(3.3)$ & 0.3 & $140(6.5)$ & 0.1 & $412(4.0)$ & 0.2 \\
\hline Hanging/strangulation/suffocation & $106(1.3)$ & 0.1 & $166(7.7)$ & 0.2 & $272(2.6)$ & 0.1 \\
\hline $\begin{array}{l}\text { Motor vehicle (e.g., car, bus, motorcycle, } \\
\text { other transport vehicle) }\end{array}$ & $63(<1.0)$ & 0.1 & $34(1.6)$ & $<0.1$ & $97(<1.0)$ & 0.1 \\
\hline Fire/burns & $46(<1.0)$ & $<0.1$ & $41(1.9)$ & $<0.1$ & $87(<1.0)$ & $<0.1$ \\
\hline Intentional neglect & $14(<1.0)$ & - & $26(1.2)$ & $<0.1$ & $40(<1.0)$ & $<0.1$ \\
\hline Poisoning & $21(<1.0)$ & $<0.1$ & $19(<1.0)$ & - & $40(<1.0)$ & $<0.1$ \\
\hline Fall & $20(<1.0)$ & $<0.1$ & $3(<1.0)$ & - & $23(<1.0)$ & $<0.1$ \\
\hline Drowning & $8(<1.0)$ & - & $8(<1.0)$ & - & $16(<1.0)$ & - \\
\hline Other (single method) & $29(<1.0)$ & - & $23(1.1)$ & - & $52(<1.0)$ & - \\
\hline Unknown & $104(1.3)$ & - & $49(2.3)$ & - & $153(1.5)$ & - \\
\hline \multicolumn{7}{|l|}{ Location } \\
\hline House/apartment & $3,402(41.6)$ & 3.7 & $1,490(69.3)$ & 1.6 & $4,892(47.3)$ & 2.6 \\
\hline Street/highway & $2,306(28.2)$ & 2.5 & $192(8.9)$ & 0.2 & $2,498(24.2)$ & 1.3 \\
\hline Motor vehicle & $788(9.6)$ & 0.9 & $144(6.7)$ & 0.2 & $932(9.0)$ & 0.5 \\
\hline Parking lot/public garage/public transport & $390(4.8)$ & 0.4 & $36(1.7)$ & $<0.1$ & $426(4.1)$ & 0.2 \\
\hline Commercial/retail area & 279 (3.4) & 0.3 & $28(1.3)$ & $<0.1$ & $307(3.0)$ & 0.2 \\
\hline Natural area & 155 (1.9) & 0.2 & $54(2.5)$ & 0.1 & $209(2.0)$ & 0.1 \\
\hline Other location ${ }^{\dagger \dagger}$ & $596(7.3)$ & - & $143(6.7)$ & - & $739(7.1)$ & - \\
\hline Unknown & $270(3.3)$ & - & $62(2.9)$ & - & $333(3.2)$ & - \\
\hline
\end{tabular}

See table footnotes on next page. 
TABLE 4. (Continued) Number, percentage, ${ }^{*}$ and rate ${ }^{\dagger}$ of homicides, by decedent's sex, age group, race/ethnicity, method used, location in which injury occurred, and victim-suspect relationship — National Violent Death Reporting System, 32 states, 82016

\begin{tabular}{|c|c|c|c|c|c|c|}
\hline \multirow[b]{2}{*}{ Characteristic } & \multicolumn{2}{|c|}{ Male } & \multicolumn{2}{|c|}{ Female } & \multicolumn{2}{|c|}{ Total } \\
\hline & No. (\%) & Rate & No. (\%) & Rate & No. $(\%)$ & Rate \\
\hline \multicolumn{7}{|l|}{ Relationship of victim to suspect ${ }^{\S \S}$} \\
\hline Acquaintance/friend & $1,009(35.2)$ & 1.1 & $153(10.5)$ & 0.2 & $1,162(26.9)$ & 0.6 \\
\hline Spouse/intimate partner (current or former) & $215(7.5)$ & 0.2 & $727(50.1)$ & 0.8 & $942(21.8)$ & 0.5 \\
\hline Other person known to victim & $519(18.1)$ & - & $104(7.2)$ & - & $623(14.4)$ & - \\
\hline Stranger & $460(16.1)$ & 0.5 & $99(6.8)$ & 0.1 & $559(13.0)$ & 0.3 \\
\hline Child & $165(5.8)$ & 0.2 & $131(9.0)$ & 0.1 & 296 (6.9) & 0.2 \\
\hline Other relative & $189(6.6)$ & - & $72(5.0)$ & - & $261(6.0)$ & - \\
\hline Parent & $117(4.1)$ & 0.1 & $119(8.2)$ & 0.1 & $236(5.5)$ & 0.1 \\
\hline Rival gang member & 109 (3.8) & 0.1 & $4(<1.0)$ & - & $113(2.6)$ & 0.1 \\
\hline Other intimate partner involvement ${ }^{\text {Iq }}$ & $60(2.1)$ & - & $41(2.8)$ & - & $101(2.3)$ & - \\
\hline $\begin{array}{l}\text { Victim was law enforcement officer injured in the line } \\
\text { of duty }\end{array}$ & $22(<1.0)$ & $<0.1$ & $1(<1.0)$ & - & $23(<1.0)$ & $<0.1$ \\
\hline Total & $8,186(100)$ & 8.9 & $2,149(100)$ & 2.3 & $10,336(100)$ & 5.5 \\
\hline
\end{tabular}

* Percentages might not total $100 \%$ due to rounding. Sex was unknown for one decedent.

† Per 100,000 population.

$\S$ Alaska, Arizona, Colorado, Connecticut, Georgia, Hawaii, Illinois, Indiana, lowa, Kansas, Kentucky, Maine, Maryland, Massachusetts, Michigan, Minnesota, New Hampshire, New Jersey, New Mexico, New York, North Carolina, Ohio, Oklahoma, Oregon, Pennsylvania, Rhode Island, South Carolina, Utah, Vermont, Virginia, Washington, and Wisconsin. Illinois, Pennsylvania, and Washington collected data on $\geq 80 \%$ of violent deaths in the state, in accordance with requirements under which the state was funded. Denominators for the rates for these three states represent only the populations of the counties from which the data were collected.

" Rates not reported when number of decedents is $<20$ or when characteristic response is "other" or "unknown."

** Includes persons of any race.

${ }^{+\dagger}$ Other location includes (in descending order): bar/nightclub, hotel/motel, park/playground/sports or athletic area, abandoned house/building/warehouse, jail/ prison, hospital or medical facility, supervised residential facility, office building, industrial or construction area, preschool/school/college/school bus, farm, cemetery/ graveyard/other burial ground, railroad tracks, synagogue/church/temple, bridge, and other unspecified location.

$\S \S$ Percentage is based on the number of homicide decedents with a known victim-suspect relationship ( $\mathrm{n}=4,316 ; 2,865$ male and 1,451 female); victim-suspect relationship was unknown for 6,020 decedents.

กศ Death was related to intimate partner violence but not between the intimate partners (e.g., a child killed by mother's boyfriend). 
TABLE 5. Number* and percentage ${ }^{\dagger}$ of homicides, by precipitating circumstances and decedent's sex - National Violent Death Reporting System, 32 states, ${ }^{\S} 2016$

\begin{tabular}{|c|c|c|c|}
\hline & Male & Female & Total \\
\hline Precipitating circumstance & No. (\%) & No. (\%) & No. (\%) \\
\hline \multicolumn{4}{|l|}{ Mental health/Substance abuse } \\
\hline Substance abuse problem (excludes alcohol) & $748(11.8)$ & $200(10.7)$ & $948(11.6)$ \\
\hline Current diagnosed mental health problem & $274(4.3)$ & $160(8.6)$ & $434(5.3)$ \\
\hline Alcohol problem & 244 (3.9) & $73(3.9)$ & 317 (3.9) \\
\hline History of ever being treated for a mental health problem & $183(2.9)$ & $119(6.4)$ & $302(3.7)$ \\
\hline Current mental health treatment & $91(1.4)$ & $77(4.1)$ & $168(2.1)$ \\
\hline Current depressed mood & $28(<1.0)$ & $27(1.4)$ & $55(<1.0)$ \\
\hline Other addiction (e.g., gambling, sex) & $13(<1.0)$ & $2(<1.0)$ & $15(<1.0)$ \\
\hline \multicolumn{4}{|l|}{ Interpersonal } \\
\hline Intimate partner violence-related & $474(7.5)$ & $806(43.2)$ & $1,280(15.6)$ \\
\hline Family relationship problem & $251(4.0)$ & $163(8.7)$ & $414(5.1)$ \\
\hline Other relationship problem (nonintimate) & $338(5.3)$ & $68(3.6)$ & $406(5.0)$ \\
\hline Jealousy (lovers' triangle) & $144(2.3)$ & $74(4.0)$ & $218(2.7)$ \\
\hline Victim of interpersonal violence during past month & $66(1.0)$ & $101(5.4)$ & $167(2.0)$ \\
\hline Perpetrator of interpersonal violence during past month & $88(1.4)$ & $6(<1.0)$ & $94(1.1)$ \\
\hline \multicolumn{4}{|l|}{ Life stressor } \\
\hline Argument or conflict & $2,096(33.1)$ & $536(28.7)$ & $2,632(32.1)$ \\
\hline Physical fight (two persons, not a brawl) & $925(14.6)$ & $138(7.4)$ & $1,063(13.0)$ \\
\hline Crisis during previous or upcoming 2 weeks & $493(7.8)$ & $215(11.5)$ & $708(8.6)$ \\
\hline History of child abuse/neglect & $62(<1.0)$ & $49(2.6)$ & $111(1.4)$ \\
\hline \multicolumn{4}{|l|}{ Crime and criminal activity } \\
\hline Precipitated by another crime & $2,088(33.0)$ & $450(24.1)$ & $2,538(31.0)$ \\
\hline Crime in progress & $1,212(58.0)$ & $224(49.8)$ & $1,436(56.6)$ \\
\hline Drug involvement & $876(13.8)$ & $108(5.8)$ & $984(12.0)$ \\
\hline Gang related & $545(8.6)$ & $60(3.2)$ & $605(7.4)$ \\
\hline Terrorist attack & $0(0.0)$ & $0(0.0)$ & $0(0.0)$ \\
\hline \multicolumn{4}{|l|}{ Homicide event } \\
\hline Drive-by shooting & $630(10.0)$ & $76(4.1)$ & $706(8.6)$ \\
\hline Walk-by assault & $491(7.8)$ & $37(2.0)$ & $528(6.4)$ \\
\hline Victim used a weapon & $438(6.9)$ & $27(1.4)$ & $465(5.7)$ \\
\hline Caretaker abuse/neglect led to death & $186(2.9)$ & $192(10.3)$ & $378(4.6)$ \\
\hline Mentally ill suspect & $125(2.0)$ & $131(7.0)$ & $256(3.1)$ \\
\hline Random violence & $192(3.0)$ & $48(2.6)$ & $240(2.9)$ \\
\hline Justifiable self-defense & $189(3.0)$ & $6(<1.0)$ & $195(2.4)$ \\
\hline Brawl & $166(2.6)$ & $12(<1.0)$ & $178(2.2)$ \\
\hline Victim was a bystander & $102(1.6)$ & $73(3.9)$ & $175(2.1)$ \\
\hline Victim was an intervener assisting a crime victim & $74(1.2)$ & $14(<1.0)$ & $88(1.1)$ \\
\hline Stalking & $18(<1.0)$ & $28(1.5)$ & $46(<1.0)$ \\
\hline Victim was a police officer on duty & $36(<1.0)$ & $1(<1.0)$ & $37(<1.0)$ \\
\hline Prostitution & $14(<1.0)$ & $22(1.2)$ & $36(<1.0)$ \\
\hline Mercy killing & $2(<1.0)$ & $12(<1.0)$ & $14(<1.0)$ \\
\hline Hate crime & $8(<1.0)$ & $0(0.0)$ & $8(<1.0)$ \\
\hline Total $^{* *}$ & $6,326(77.3)$ & $1,866(86.8)$ & $8,192(79.3)$ \\
\hline
\end{tabular}

* Includes homicides with one or more precipitating circumstances. Total numbers do not equal the sums of the columns because more than one circumstance could have been present per decedent. Circumstances were unknown for 2,144 decedents (1,860 males and 283 females; sex was unknown for one decedent).

+ Denominator includes those homicides with one or more precipitating circumstances. The sum of percentages in columns exceeds $100 \%$ because more than one circumstance could have been present per decedent.

$\S$ Alaska, Arizona, Colorado, Connecticut, Georgia, Hawaii, Illinois, Indiana, lowa, Kansas, Kentucky, Maine, Maryland, Massachusetts, Michigan, Minnesota, New Hampshire, New Jersey, New Mexico, New York, North Carolina, Ohio, Oklahoma, Oregon, Pennsylvania, Rhode Island, South Carolina, Utah, Vermont, Virginia, Washington, and Wisconsin. Illinois, Pennsylvania, and Washington collected data on $\geq 80 \%$ of violent deaths in the state, in accordance with requirements under which the state was funded.

9 Denominator includes those decedents involved in an incident that was precipitated by another crime.

** Total no. of homicide decedents $=10,336$ (8,186 males and 2,149 females). 
TABLE 6. Number, percentage, ${ }^{*}$ and rate ${ }^{\dagger}$ of legal intervention ${ }^{\S}$ deaths, by decedent's sex, age group, race/ethnicity, method used, and location in which injury occurred - National Violent Death Reporting System, 32 states, ${ }^{\mathbb{\imath}} 2016$

\begin{tabular}{|c|c|c|c|c|c|c|}
\hline \multirow[b]{2}{*}{ Characteristic } & \multicolumn{2}{|c|}{ Male } & \multicolumn{2}{|c|}{ Female } & \multicolumn{2}{|c|}{ Total } \\
\hline & No. (\%) & Rate & No. (\%) & Rate & No. (\%) & Rate \\
\hline \multicolumn{7}{|l|}{ Age group (yrs) } \\
\hline$<1$ & $0(0.0)$ & - ** & $0(0.0)$ & - & $0(0.0)$ & - \\
\hline $1-4$ & $0(0.0)$ & - & $0(0.0)$ & - & $0(0.0)$ & - \\
\hline $5-9$ & $0(0.0)$ & - & $0(0.0)$ & - & $0(0.0)$ & - \\
\hline $10-14$ & $1(<1.0)$ & - & $0(0.0)$ & - & $1(<1.0)$ & - \\
\hline 15-19 & $20(4.1)$ & 0.3 & $0(0.0)$ & - & $20(3.9)$ & 0.2 \\
\hline $20-24$ & $57(11.6)$ & 0.9 & $4(17.4)$ & - & $61(11.8)$ & 0.5 \\
\hline $25-29$ & $79(16.1)$ & 1.2 & $4(17.4)$ & - & $83(16.1)$ & 0.6 \\
\hline $30-34$ & $84(17.1)$ & 1.3 & $4(17.4)$ & - & $88(17.1)$ & 0.7 \\
\hline $35-44$ & $127(25.8)$ & 1.1 & $6(26.1)$ & - & $133(25.8)$ & 0.6 \\
\hline $45-54$ & $64(13.0)$ & 0.5 & $2(8.7)$ & - & $66(12.8)$ & 0.3 \\
\hline $55-64$ & $48(9.8)$ & 0.4 & $2(8.7)$ & - & $50(9.7)$ & 0.2 \\
\hline $65-74$ & $10(2.0)$ & - & $1(4.3)$ & - & $11(2.1)$ & - \\
\hline $75-84$ & $2(<1.0)$ & - & $0(0.0)$ & - & $2(<1.0)$ & - \\
\hline$\geq 85$ & $0(0.0)$ & - & $0(0.0)$ & - & $0(0.0)$ & - \\
\hline Unknown & $0(0.0)$ & - & $0(0.0)$ & - & $0(0.0)$ & - \\
\hline \multicolumn{7}{|l|}{ Race/Ethnicity } \\
\hline White, non-Hispanic & $246(50.0)$ & 0.4 & $11(47.8)$ & - & $257(49.9)$ & 0.2 \\
\hline Black, non-Hispanic & $136(27.6)$ & 1.1 & $6(26.1)$ & - & $142(27.6)$ & 0.6 \\
\hline American Indian/Alaska Native, non-Hispanic & $19(3.9)$ & - & $4(17.4)$ & - & $23(4.5)$ & 1.2 \\
\hline Asian/Pacific Islander & $14(2.8)$ & - & $0(0.0)$ & - & $14(2.7)$ & - \\
\hline Hispanic ${ }^{\dagger \dagger}$ & $76(15.4)$ & 0.7 & $2(8.7)$ & - & $78(15.1)$ & 0.3 \\
\hline Other & $1(<1.0)$ & - & $0(0.0)$ & - & $1(<1.0)$ & - \\
\hline Unknown & $0(0.0)$ & - & $0(0.0)$ & - & $0(0.0)$ & - \\
\hline \multicolumn{7}{|l|}{ Method } \\
\hline Firearm & $472(95.9)$ & 0.5 & $23(100)$ & $<0.1$ & $495(96.1)$ & 0.3 \\
\hline $\begin{array}{l}\text { Motor vehicle (e.g., car, bus, motorcycle, } \\
\text { other transport vehicle) }\end{array}$ & $7(1.4)$ & - & $0(0.0)$ & - & $7(1.4)$ & - \\
\hline Drowning & $2(<1.0)$ & - & $0(0.0)$ & - & $2(<1.0)$ & - \\
\hline Hanging/strangulation/suffocation & $1(<1.0)$ & - & $0(0.0)$ & - & $1(<1.0)$ & - \\
\hline Poisoning & $1(<1.0)$ & - & $0(0.0)$ & - & $1(<1.0)$ & - \\
\hline Blunt instrument & $0(0.0)$ & - & $0(0.0)$ & - & $0(0.0)$ & - \\
\hline Fall & $0(0.0)$ & - & $0(0.0)$ & - & $0(0.0)$ & - \\
\hline Fire/burns & $0(0.0)$ & - & $0(0.0)$ & - & $0(0.0)$ & - \\
\hline Intentional neglect & $0(0.0)$ & - & $0(0.0)$ & - & $0(0.0)$ & - \\
\hline Personal weapons (e.g., hands, feet, fists) & $0(0.0)$ & - & $0(0.0)$ & - & $0(0.0)$ & - \\
\hline Sharp instrument & $0(0.0)$ & - & $0(0.0)$ & - & $0(0.0)$ & - \\
\hline Other (single method) & $3(<1.0)$ & - & $0(0.0)$ & - & $3(<1.0)$ & - \\
\hline Unknown & $6(1.2)$ & - & $0(0.0)$ & - & $6(1.2)$ & - \\
\hline \multicolumn{7}{|l|}{ Location } \\
\hline House/apartment & $187(38.0)$ & 0.2 & $11(47.8)$ & - & $198(38.4)$ & 0.1 \\
\hline Street/highway & $143(29.1)$ & 0.2 & $3(13.0)$ & - & $146(28.3)$ & 0.1 \\
\hline Motor vehicle & $47(9.6)$ & 0.1 & $2(8.7)$ & - & $49(9.5)$ & $<0.1$ \\
\hline Parking lot/public garage/public transport & $28(5.7)$ & $<0.1$ & $2(8.7)$ & - & $30(5.8)$ & $<0.1$ \\
\hline Commercial/retail area & $23(4.7)$ & $<0.1$ & $3(13.0)$ & - & $26(5.0)$ & $<0.1$ \\
\hline Other location $\S^{\S}$ & $51(10.4)$ & - & $2(8.7)$ & - & $53(10.3)$ & - \\
\hline Unknown & $13(2.6)$ & - & $0(0.0)$ & - & $13(2.5)$ & - \\
\hline Total & $492(100)$ & 0.5 & $23(100)$ & $<0.1$ & $515(100)$ & 0.3 \\
\hline
\end{tabular}

* Percentages might not total $100 \%$ due to rounding.

+ Per 100,000 population.

$\S$ The term legal intervention does not denote the lawfulness or legality of the circumstances surrounding the death.

I Alaska, Arizona, Colorado, Connecticut, Georgia, Hawaii, Illinois, Indiana, lowa, Kansas, Kentucky, Maine, Maryland, Massachusetts, Michigan, Minnesota, New Hampshire, New Jersey, New Mexico, New York, North Carolina, Ohio, Oklahoma, Oregon, Pennsylvania, Rhode Island, South Carolina, Utah, Vermont, Virginia, Washington, and Wisconsin. Illinois, Pennsylvania, and Washington collected data on $\geq 80 \%$ of violent deaths in the state, in accordance with requirements under which the state was funded. Denominators for the rates for these three states represent only the populations of the counties from which the data were collected.

** Rates not reported when number of decedents is $<20$ or when characteristic response is "other" or "unknown."

t+ Includes persons of any race.

$\S \S$ Other location includes (in descending order): natural area, hotel/motel, park/playground/sports or athletic area, bar/nightclub, hospital or medical facility, preschool/ school/college/school bus, abandoned house/building/warehouse, bridge, farm, industrial or construction area, jail/prison, office building, and other unspecified location. 
TABLE 7. Number* and percentage† of legal intervention ${ }^{\S}$ deaths, by precipitating circumstances and decedent's sex — National Violent Death Reporting System, 32 states, ${ }^{\mathbb{N}} 2016$

\begin{tabular}{|c|c|c|c|}
\hline & Male & Female & Total \\
\hline Precipitating circumstance & No. (\%) & No. (\%) & No. (\%) \\
\hline \multicolumn{4}{|l|}{ Mental health/Substance abuse } \\
\hline Substance abuse problem (excludes alcohol) & $126(26.1)$ & $4(18.2)$ & $130(25.7)$ \\
\hline Current diagnosed mental health problem & $99(20.5)$ & $7(31.8)$ & $106(21.0)$ \\
\hline History of ever being treated for a mental health problem & $73(15.1)$ & $5(22.7)$ & 78 (15.4) \\
\hline Alcohol problem & $50(10.4)$ & $2(9.1)$ & $52(10.3)$ \\
\hline Current mental health treatment & $36(7.5)$ & $2(9.1)$ & $38(7.5)$ \\
\hline Current depressed mood & $29(6.0)$ & $2(9.1)$ & $31(6.1)$ \\
\hline Other addiction (e.g., gambling, sex) & $3(<1.0)$ & $0(0.0)$ & $3(<1.0)$ \\
\hline \multicolumn{4}{|l|}{ Interpersonal } \\
\hline Perpetrator of interpersonal violence during past month & $46(9.5)$ & $3(13.6)$ & $49(9.7)$ \\
\hline Intimate partner violence-related & $45(9.3)$ & $0(0.0)$ & $45(8.9)$ \\
\hline Family relationship problem & $33(6.8)$ & $2(9.1)$ & $35(6.9)$ \\
\hline Other relationship problem (nonintimate) & $15(3.1)$ & $1(4.5)$ & $16(3.2)$ \\
\hline Jealousy (lovers' triangle) & $3(<1.0)$ & $0(0.0)$ & $3(<1.0)$ \\
\hline Victim of interpersonal violence during past month & $1(<1.0)$ & $0(0.0)$ & $1(<1.0)$ \\
\hline \multicolumn{4}{|l|}{ Life stressor } \\
\hline Crisis during previous or upcoming 2 weeks & $73(15.1)$ & $4(18.2)$ & $77(15.2)$ \\
\hline Argument or conflict & 67 (13.9) & $4(18.2)$ & $71(14.1)$ \\
\hline Physical fight (two persons, not a brawl) & $38(7.9)$ & $1(4.5)$ & $39(7.7)$ \\
\hline History of child abuse/neglect & $2(<1.0)$ & $0(0.0)$ & $2(<1.0)$ \\
\hline \multicolumn{4}{|l|}{ Crime and criminal activity } \\
\hline Precipitated by another crime & $435(90.1)$ & $18(81.8)$ & $453(89.7)$ \\
\hline Crime in progress ${ }^{* *}$ & $287(66.0)$ & $11(61.1)$ & $298(65.8)$ \\
\hline Drug involvement & $28(5.8)$ & $1(4.5)$ & $29(5.7)$ \\
\hline Gang-related & $4(<1.0)$ & $0(0.0)$ & $4(<1.0)$ \\
\hline Terrorist attack & $0(0.0)$ & $0(0.0)$ & $0(0.0)$ \\
\hline \multicolumn{4}{|l|}{ Legal intervention event } \\
\hline Victim used a weapon & 352 (72.9) & $16(72.7)$ & $368(72.9)$ \\
\hline Brawl & $3(<1.0)$ & $0(0.0)$ & $3(<1.0)$ \\
\hline Prostitution & $2(<1.0)$ & $0(0.0)$ & $2(<1.0)$ \\
\hline Victim was a bystander & $1(<1.0)$ & $0(0.0)$ & $1(<1.0)$ \\
\hline Victim was a police officer on duty & $1(<1.0)$ & $0(0.0)$ & $1(<1.0)$ \\
\hline Random violence & $1(<1.0)$ & $0(0.0)$ & $1(<1.0)$ \\
\hline Stalking & $1(<1.0)$ & $0(0.0)$ & $1(<1.0)$ \\
\hline Victim was an intervener assisting a crime victim & $0(0.0)$ & $0(0.0)$ & $0(0.0)$ \\
\hline Mentally ill suspect & $0(0.0)$ & $0(0.0)$ & $0(0.0)$ \\
\hline Total ${ }^{\dagger+}$ & $483(98.2)$ & $22(95.7)$ & $505(98.1)$ \\
\hline
\end{tabular}

* Includes deaths with one or more precipitating circumstances. Total numbers do not equal the sums of the columns because more than one circumstance could have been present per decedent.

${ }^{\dagger}$ Denominator includes those deaths with one or more precipitating circumstances. The sum of percentages in columns exceed $100 \%$ because more than one circumstance could have been present per decedent.

$\S$ The term legal intervention does not denote the lawfulness or legality of the circumstances surrounding the death.

I Alaska, Arizona, Colorado, Connecticut, Georgia, Hawaii, Illinois, Indiana, lowa, Kansas, Kentucky, Maine, Maryland, Massachusetts, Michigan, Minnesota, New Hampshire, New Jersey, New Mexico, New York, North Carolina, Ohio, Oklahoma, Oregon, Pennsylvania, Rhode Island, South Carolina, Utah, Vermont, Virginia, Washington, and Wisconsin. Illinois, Pennsylvania, and Washington collected data on $\geq 80 \%$ of violent deaths in the state, in accordance with requirements under which the state was funded.

** Denominator includes those decedents involved in an incident that was precipitated by another crime.

${ }^{+\dagger}$ Circumstances were unknown for 10 decedents (nine males and one female); total no. of legal intervention deaths $=515$ (492 males and 23 females). 
TABLE 8. Number and percentage* of unintentional firearm deaths, by decedent's sex, race/ethnicity, age group, location of injury, and type of firearm - National Violent Death Reporting System, 32 states, $^{\dagger} 2016$

\begin{tabular}{lr} 
Characteristic & No. $(\%)$ \\
\hline Sex & \\
Male & $255(86.4)$ \\
Female & $40(13.6)$ \\
Race/Ethnicity & \\
White, non-Hispanic & $175(59.3)$ \\
Black, non-Hispanic & $86(29.2)$ \\
American Indian/Alaska Native, non-Hispanic & $3(1.0)$ \\
Asian/Pacific Islander & $2(<1.0)$ \\
Hispanic $\$$ & $27(9.2)$ \\
Other & $2(<1.0)$ \\
Age group (yrs) & \\
$<1$ & $0(0.0)$ \\
1-4 & $23(7.8)$ \\
$5-9$ & $5(1.7)$ \\
10-14 & $23(7.8)$ \\
15-19 & $58(19.7)$ \\
20-24 & $44(14.9)$ \\
25-29 & $28(9.5)$ \\
30-34 & $21(7.1)$ \\
35-44 & $10(3.4)$ \\
45-54 & $26(8.8)$ \\
$55-64$ & $32(10.8)$ \\
65-74 & $10(3.4)$ \\
$75-84$ & $9(3.1)$ \\
$\geq 85$ & $6(2.0)$ \\
Location & \\
House/apartment & $218(73.9)$ \\
Natural area & $26(8.8)$ \\
Motor vehicle & $13(4.4)$ \\
Street/highway & $13(4.4)$ \\
Other location & $17(5.8)$ \\
Unknown & $8(2.7)$ \\
Firearm type & \\
Handgun & $186(63.1)$ \\
Rifle & $32(10.8)$ \\
Shotgun & $25(8.5)$ \\
Other firearm & $1(<1.0)$ \\
Unknown & $51(17.3)$ \\
Total & $295(100)$ \\
\hline *Percentas &
\end{tabular}

* Percentages might not total $100 \%$ due to rounding.

† Alaska, Arizona, Colorado, Connecticut, Georgia, Hawaii, Illinois, Indiana, lowa, Kansas, Kentucky, Maine, Maryland, Massachusetts, Michigan, Minnesota, New Hampshire, New Jersey, New Mexico, New York, North Carolina, Ohio, Oklahoma, Oregon, Pennsylvania, Rhode Island, South Carolina, Utah, Vermont, Virginia, Washington, and Wisconsin. Illinois, Pennsylvania, and Washington collected data on $\geq 80 \%$ of violent deaths in the state, in accordance with requirements under which the state was funded.

$\S$ Includes persons of any race.

" Other location includes (in descending order): commercial/retail area, parking lot/public garage/public transport, abandoned house/building/warehouse, park/playground/sports or athletic area, farm, hotel/motel, and other unspecified location.
TABLE 9. Number and percentage* of unintentional firearm deaths, by contexts and circumstances of injury - National Violent Death Reporting System, 32 states, ${ }^{\dagger} 2016$

\begin{tabular}{lr}
\hline Characteristic & No. (\%) \\
\hline Context of injury & \\
Playing with gun & $93(34.7)$ \\
Showing gun to others & $33(12.3)$ \\
Cleaning gun & $27(10.1)$ \\
Hunting & $21(7.8)$ \\
Loading/unloading gun & $11(4.1)$ \\
Target shooting & $7(2.6)$ \\
Celebratory firing & $0(0.0)$ \\
Other context of injury & $71(26.5)$ \\
Circumstance of injury & \\
Unintentionally pulled trigger & $63(23.5)$ \\
Thought gun was unloaded & $24(9.0)$ \\
Thought unloaded/magazine disengaged & $16(6.0)$ \\
Gun was dropped & $15(5.6)$ \\
Gun fired due to defect or malfunction & $10(3.7)$ \\
Gun was mistaken for a toy & $8(3.0)$ \\
Gun fired while holstering/unholstering & $6(2.2)$ \\
Thought gun safety was engaged & $5(1.9)$ \\
Bullet ricocheted & $3(1.1)$ \\
Gun fired while handling safety/lock & $1(<1.0)$ \\
Other mechanism of injury & $63(23.5)$ \\
Total $\$$ & $268(90.8)$ \\
\hline
\end{tabular}

* Percentages might exceed $100 \%$ because one or more circumstances could have been known per death; therefore, number and percentage are reported when the number of deaths is $<5$ because no particular circumstance identifies a single death.

† Alaska, Arizona, Colorado, Connecticut, Georgia, Hawaii, Illinois, Indiana, lowa, Kansas, Kentucky, Maine, Maryland, Massachusetts, Michigan, Minnesota, New Hampshire, New Jersey, New Mexico, New York, North Carolina, Ohio, Oklahoma, Oregon, Pennsylvania, Rhode Island, South Carolina, Utah, Vermont, Virginia, Washington, and Wisconsin. Illinois, Pennsylvania, and Washington collected data on $\geq 80 \%$ of violent deaths in the state, in accordance with requirements under which the state was funded.

$\S$ Circumstances were unknown for 27 decedents; total no. of unintentional firearm decedents $=295$. 
TABLE 10. Number and percentage* of youth suicides by age group, sex, race/ethnicity, method used, and location in which injury occurred National Violent Death Reporting System, 32 states, ${ }^{\dagger} 2016$

\begin{tabular}{|c|c|c|c|}
\hline & $10-17$ yrs & $18-24$ yrs & Total \\
\hline Characteristic & No. (\%) & No. (\%) & No. (\%) \\
\hline \multicolumn{4}{|l|}{ Sex } \\
\hline Male & $632(69.4)$ & $2,244(81.8)$ & $2,876(78.7)$ \\
\hline Female & 279 (30.6) & $500(18.2)$ & 779 (21.3) \\
\hline \multicolumn{4}{|l|}{ Race/Ethnicity } \\
\hline White, non-Hispanic & $646(70.9)$ & $1,842(67.1)$ & $2,488(68.1)$ \\
\hline Black, non-Hispanic & $84(9.2)$ & $350(12.8)$ & 434 (11.9) \\
\hline American Indian/Alaska Native, non-Hispanic & $30(3.3)$ & $99(3.6)$ & $129(3.5)$ \\
\hline Asian/Pacific Islander & $37(4.1)$ & $151(5.5)$ & $188(5.1)$ \\
\hline Hispanic ${ }^{\S}$ & 108 (11.9) & $289(10.5)$ & 397 (10.9) \\
\hline Other & $6(<1.0)$ & $13(<1.0)$ & $19(<1.0)$ \\
\hline \multicolumn{4}{|l|}{ Method } \\
\hline Firearm & $368(40.4)$ & $1,267(46.2)$ & $1,635(44.7)$ \\
\hline Hanging/strangulation/suffocation & $449(49.3)$ & $1,025(37.4)$ & $1,474(40.3)$ \\
\hline Poisoning & $39(4.3)$ & $191(7.0)$ & $230(6.3)$ \\
\hline Fall & $23(2.5)$ & $103(3.8)$ & $126(3.4)$ \\
\hline Motor vehicle (e.g., car, bus, motorcycle, other transport vehicle) & $23(2.5)$ & $82(3.0)$ & $105(2.9)$ \\
\hline Drowning & $4(<1.0)$ & $31(1.1)$ & $35(<1.0)$ \\
\hline Sharp instrument & $1(<1.0)$ & $23(<1.0)$ & $24(<1.0)$ \\
\hline Fire/burns & $1(<1.0)$ & $13(<1.0)$ & $14(<1.0)$ \\
\hline Blunt instrument & $0(0.0)$ & $2(<1.0)$ & $2(<1.0)$ \\
\hline Other (single method) & $2(<1.0)$ & $4(<1.0)$ & $6(<1.0)$ \\
\hline Unknown & $1(<1.0)$ & $3(<1.0)$ & $4(<1.0)$ \\
\hline \multicolumn{4}{|l|}{ Location } \\
\hline House/apartment & $737(80.9)$ & $1,801(65.6)$ & $2,538(69.4)$ \\
\hline Natural area & $50(5.5)$ & $203(7.4)$ & $253(6.9)$ \\
\hline Motor vehicle & $15(1.6)$ & $168(6.1)$ & $183(5.0)$ \\
\hline Street/highway & $12(1.3)$ & $89(3.2)$ & $101(2.8)$ \\
\hline Park/playground/sports or athletic area & $16(1.8)$ & $74(2.7)$ & $90(2.5)$ \\
\hline Parking lot/public garage/public transport & $9(<1.0)$ & $50(1.8)$ & $59(1.6)$ \\
\hline Preschool/school/college/school bus & $11(1.2)$ & $45(1.6)$ & $56(1.5)$ \\
\hline Other location? & $48(5.3)$ & $284(10.3)$ & $332(9.1)$ \\
\hline Unknown & $13(1.4)$ & $30(1.1)$ & $43(1.2)$ \\
\hline Total & $911(100)$ & $2,744(100)$ & $3,655(100)$ \\
\hline
\end{tabular}

* Percentages might not total $100 \%$ due to rounding.

† Alaska, Arizona, Colorado, Connecticut, Georgia, Hawaii, Illinois, Indiana, lowa, Kansas, Kentucky, Maine, Maryland, Massachusetts, Michigan, Minnesota, New Hampshire, New Jersey, New Mexico, New York, North Carolina, Ohio, Oklahoma, Oregon, Pennsylvania, Rhode Island, South Carolina, Utah, Vermont, Virginia, Washington, and Wisconsin. Illinois, Pennsylvania, and Washington collected data on $\geq 80 \%$ of violent deaths in the state, in accordance with requirements under which the state was funded.

$\S$ Includes persons of any race.

" Other location includes (in descending order): railroad tracks, bridge, hotel/motel, jail/prison, commercial/retail area, farm, supervised residential facility, hospital or medical facility, cemetery/graveyard/other burial ground, office building, abandoned house/building/warehouse, industrial or construction area, synagogue/ church/temple, bar/nightclub, and other unspecified location. 
TABLE 11. Number* and percentage of youth suicide decedents who were tested for alcohol and drugs whose results were positive, ${ }^{\dagger}$ by age group and toxicology variables — National Violent Death Reporting System, 32 states, $\$ 2016$

\begin{tabular}{|c|c|c|c|c|}
\hline \multirow[b]{3}{*}{ Toxicology variable } & \multicolumn{2}{|c|}{$10-17$ yrs } & \multicolumn{2}{|c|}{$18-24$ yrs } \\
\hline & Tested & Positive & Tested & Positive \\
\hline & No. (\%) & No. (\%) & No. (\%) & No. (\%) \\
\hline$B A C^{q}$ & $463(50.8)$ & $50(10.8)$ & $1,460(53.2)$ & $570(39.0)$ \\
\hline Alcohol $<0.08 \mathrm{~g} / \mathrm{dL}$ & & & $28(56.0)$ & $178(31.2)$ \\
\hline Alcohol $\geq 0.08 \mathrm{~g} / \mathrm{dL}$ & & & $18(36.0)$ & $369(64.7)$ \\
\hline Alcohol positive, level unknown & & & $4(8.0)$ & $23(4.0)$ \\
\hline Amphetamines & $382(41.9)$ & $38(9.9)$ & $1,057(38.5)$ & $143(13.5)$ \\
\hline Anticonvulsants & $204(22.4)$ & $15(7.4)$ & $550(20.0)$ & $51(9.3)$ \\
\hline Antidepressants & $258(28.3)$ & $73(28.3)$ & $668(24.3)$ & $152(22.8)$ \\
\hline Antipsychotics & $217(23.8)$ & $5(2.3)$ & $602(21.9)$ & $24(4.0)$ \\
\hline Barbiturates & $313(34.4)$ & $3(<1.0)$ & $868(31.6)$ & $9(1.0)$ \\
\hline Benzodiazepines & $358(39.3)$ & $31(8.7)$ & $1,047(38.2)$ & $206(19.7)$ \\
\hline Carbon monoxide & $57(6.3)$ & $3(5.3)$ & $186(6.8)$ & $34(18.3)$ \\
\hline Cocaine & $362(39.7)$ & $2(<1.0)$ & $1,092(39.8)$ & $85(7.8)$ \\
\hline Marijuana & $335(36.8)$ & $83(24.8)$ & $1,006(36.7)$ & $431(42.8)$ \\
\hline Muscle relaxants & $235(25.8)$ & $2(<1.0)$ & $610(22.2)$ & $17(2.8)$ \\
\hline Opioids & $377(41.4)$ & $23(6.1)$ & $1,108(40.4)$ & $150(13.5)$ \\
\hline Other drugs/substances** & $136(14.9)$ & $88(64.7)$ & $514(18.7)$ & $367(71.4)$ \\
\hline
\end{tabular}

Abbreviation: $\mathrm{BAC}=$ blood alcohol concentration.

${ }^{*}$ No. of youth suicide decedents aged $10-17$ years $=911$ and aged $18-24$ years $=2,744$.

† Percentage is of decedents tested for toxicology variable.

$\S$ Alaska, Arizona, Colorado, Connecticut, Georgia, Hawaii, Illinois, Indiana, lowa, Kansas, Kentucky, Maine, Maryland, Massachusetts, Michigan, Minnesota, New Hampshire, New Jersey, New Mexico, New York, North Carolina, Ohio, Oklahoma, Oregon, Pennsylvania, Rhode Island, South Carolina, Utah, Vermont, Virginia, Washington, and Wisconsin. Illinois, Pennsylvania, and Washington collected data on $\geq 80 \%$ of violent deaths in the state, in accordance with requirements under which the state was funded.

I $B A C \geq 0.08 \mathrm{~g} / \mathrm{dL}$ is over the legal limit for impaired driving.

** Other drugs/substances indicated if any results were positive; levels for these drugs/substances are not measured. 
TABLE 12. Number* and percentage ${ }^{\dagger}$ of youth suicides, by precipitating circumstances and decedent's age group — National Violent Death Reporting System, 32 states, $\$ 2016$

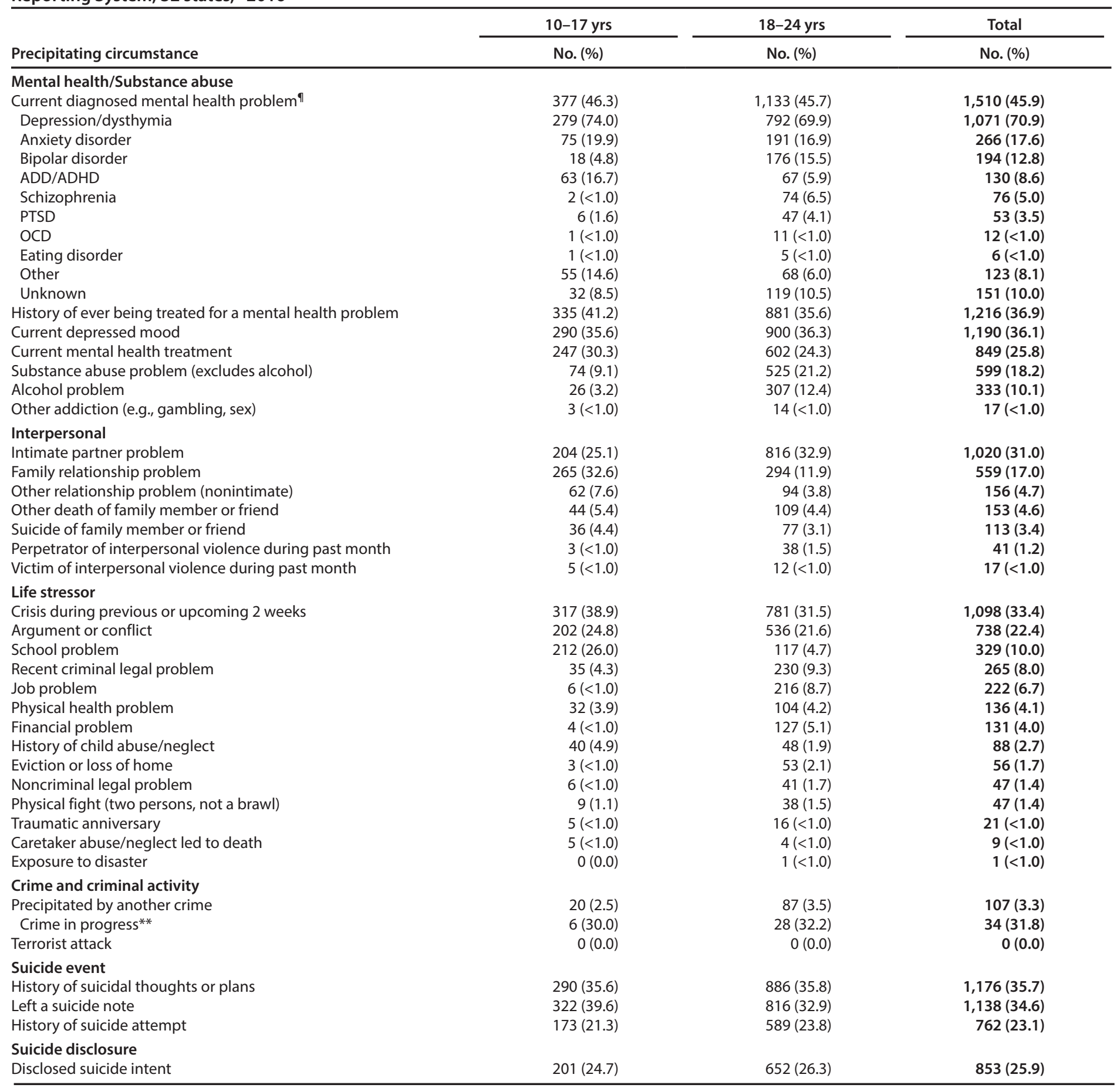

See table footnotes on next page. 
TABLE 12. (Continued) Number* and percentage ${ }^{\dagger}$ of youth suicides, by precipitating circumstances and decedent's age group - National Violent Death Reporting System, 32 states, $\$ 2016$

\begin{tabular}{|c|c|c|c|}
\hline & $10-17$ yrs & $18-24$ yrs & Total \\
\hline Precipitating circumstance & No. (\%) & No. (\%) & No. (\%) \\
\hline \multicolumn{4}{|l|}{ Disclosed intent to whom ${ }^{\dagger \dagger}$} \\
\hline Previous or current intimate partner & $38(18.9)$ & $232(35.6)$ & $270(31.7)$ \\
\hline Other family member & $57(28.4)$ & $194(29.8)$ & $251(29.4)$ \\
\hline Friend/colleague & $65(32.3)$ & $120(18.4)$ & $185(21.7)$ \\
\hline Health care worker & $7(3.5)$ & $14(2.1)$ & $21(2.5)$ \\
\hline Other person & $22(10.9)$ & $57(8.7)$ & $79(9.3)$ \\
\hline Unknown & $12(6.0)$ & $35(5.4)$ & $47(5.5)$ \\
\hline Total§§ & $814(89.4)$ & $2,478(90.3)$ & $3,292(90.1)$ \\
\hline
\end{tabular}

Abbreviations: $\mathrm{ADD} / \mathrm{ADHD}=$ attention deficit disorder/attention deficit hyperactivity disorder; $\mathrm{OCD}=$ obsessive-compulsive disorder; $\mathrm{PTSD}=$ posttraumatic stress disorder.

* Includes suicides with one or more precipitating circumstances. More than one circumstance could have been present per decedent.

${ }^{\dagger}$ Denominator includes those suicides with one or more precipitating circumstances. The sum of percentages in columns exceeds $100 \%$ because more than one circumstance could have been present per decedent.

$\S$ Alaska, Arizona, Colorado, Connecticut, Georgia, Hawaii, Illinois, Indiana, lowa, Kansas, Kentucky, Maine, Maryland, Massachusetts, Michigan, Minnesota, New Hampshire, New Jersey, New Mexico, New York, North Carolina, Ohio, Oklahoma, Oregon, Pennsylvania, Rhode Island, South Carolina, Utah, Vermont, Virginia, Washington, and Wisconsin. Illinois, Pennsylvania, and Washington collected data on $\geq 80 \%$ of violent deaths in the state, in accordance with requirements under which the state was funded.

I Includes decedents with one or more diagnosed current mental health problems; therefore, sums of percentages for the diagnosed conditions exceed $100 \%$. Denominator includes the number of decedents with one or more current diagnosed mental health problems.

** Denominator includes those decedents involved in an incident that was precipitated by another crime.

t† Denominator includes decedents who disclosed intent.

$\S \S$ Circumstances were unknown for 363 decedents ( 97 aged 10-17 years and 266 aged 18-24 years); total no. of youth suicide decedents $=3,655$ ( 911 aged $10-17$ years and 2,744 aged 18-24 years). 

The Morbidity and Mortality Weekly Report (MMWR) Series is prepared by the Centers for Disease Control and Prevention (CDC) and is available free of charge in electronic format. To receive an electronic copy each week, visit MMWR at https://www.cdc.gov/mmwr/index.html.

Readers who have difficulty accessing this PDF file may access the HTML file at https://www.cdc.gov/mmwr/volumes/68/ss/ss6809a1.htm?s_ cid=ss6809a1_w. Address all inquiries about the $M M W R$ Series, including material to be considered for publication, to Executive Editor, $M M W R$ Series, Mailstop E-90, CDC, 1600 Clifton Rd., N.E., Atlanta, GA 30329-4027 or to mmwrq@cdc.gov.

All material in the MMWR Series is in the public domain and may be used and reprinted without permission; citation as to source, however, is appreciated. MMWR and Morbidity and Mortality Weekly Report are service marks of the U.S. Department of Health and Human Services.

Use of trade names and commercial sources is for identification only and does not imply endorsement by the U.S. Department of Health and Human Services.

References to non-CDC sites on the Internet are provided as a service to $M M W R$ readers and do not constitute or imply endorsement of these organizations or their programs by CDC or the U.S. Department of Health and Human Services. CDC is not responsible for the content of these sites. URL addresses listed in $M M W R$ were current as of the date of publication.

ISSN: 0149-2195 (Print) 